\title{
Soft factors from classical scattering on the Reissner-Nordström spacetime
}

\author{
Karan Fernandes $\odot^{*}$ \\ Harish-Chandra Research Institute, Chhatnag Road, Jhusi, Prayagraj 211019, India \\ Arpita Mitra $^{\dagger}$ \\ Department of Physics, Indian Institute of Science Education and Research (IISER) Bhopal, \\ Bhopal 462066, India
}

(Received 20 July 2020; accepted 8 October 2020; published 12 November 2020)

\begin{abstract}
We consider perturbations of the four-dimensional Reissner-Nordström spacetime induced by the probe scattering of a point particle with charge and mass moving on an unbound trajectory with an asymptotically large velocity. The resulting classical radiative solutions are the gravitational and electromagnetic bremsstrahlung. We use these classical solutions to derive the universal photon and graviton soft factor contributions at the tree level, which have the same form as noted in the literature. The soft factor expressions enable us to investigate the tail contribution to the memory effect in late time gravitational and electromagnetic waveforms. We find that generically, the contribution from the charge dominates that from the mass in the late time radiation.
\end{abstract}

DOI: 10.1103/PhysRevD.102.105015

\section{INTRODUCTION}

The radiation emitted by accelerated particles (bremsstrahlung) on curved spacetimes cover an important class of scattering problems in classical general relativity. Solutions include the electromagnetic radiation emitted by point charges [1-3] and the gravitational radiation emitted by point masses [4,5] on the Schwarzschild background. One of the reasons for interest in these solutions concerns the power spectrum of the emitted radiation, which gives an estimate on their energy and angular distribution. An interesting regime in this spectrum lies in the soft limit, $\omega \rightarrow 0$. As this limit involves long wavelengths, the power spectrum result can be expected to be independent of specific details concerning the internal structure of the bodies and the nature of their scattering. This was demonstrated in [6], where the zero-frequency limit of gravitational bremsstrahlung was considered in the case of distant encounters as well as head-on collisions. The soft limit result derived by Smarr at the linearized level agrees with postlinear results and the full nonlinear numerical result for the head-on collision of equal mass black holes [7-11].

\footnotetext{
*karanfernandes@hri.res.in

arpitam@iiserb.ac.in
}

Published by the American Physical Society under the terms of the Creative Commons Attribution 4.0 International license. Further distribution of this work must maintain attribution to the author(s) and the published article's title, journal citation, and DOI. Funded by SCOAP ${ }^{3}$.
The soft limit is also of particular importance in the context of soft theorems on asymptotically flat spacetimes. Soft theorems relate scattering amplitudes involving soft particles with amplitudes without soft particles through a soft factor. The leading pole contribution to the soft photon and graviton factors [12,13] and soft photon expansions of the S-matrix [14-16] pertained to infrared properties of quantum field theories. Soft theorems are now known to be more generally related with asymptotic symmetries on aymptotically flat spacetimes [17-29]. The universal contributions in soft factors result as a consequence of invariance under $U(1)$ transformations in the case of Abelian gauge theories and diffeomorphisms in the case of gravitational theories. There have been many recent developments of soft theorems concerning subleading contributions [30-40], loop corrections [41-51], and multiple soft particles [52-63]. The universal contributions in soft factors and their connection with asymptotic symmetries have in particular led to several investigations concerning gravitational waves and the memory effect [64-72].

This paper is motivated by the results of [70], where it was shown that universal contributions in the photon and graviton soft factors in any spacetime dimensions can be derived from classical electromagnetic and gravitational scattering processes. The use of classical scattering processes in determining soft factors is particularly relevant in four spacetime dimensions, where the S-matrix suffers from infrared divergences. In [71], the soft factors were shown to develop logarithmic terms in the classical limit of soft theorems in four spacetime dimensions, arising from 
the long range interactions in electromagnetism and gravity. Their analysis also demonstrated that the classical gravitational bremsstrahlung from a point mass on the Schwarzschild spacetime [4] satisfies the soft graviton theorem in four spacetime dimensions. Furthermore, the comparison with [4] also revealed the presence of an overall phase, which influences the shape of the gravitational waveform. This additional phase does not follow from taking the classical limit of soft theorems and can only be inferred in comparison with classical results. The soft graviton factor involving the phase correction has been used to identify their effect on gravitational waves [72]. In addition to the gravitational memory effect which follows from the leading contribution, the logarithmic terms present in the subleading contribution of the soft factor imply the presence of a tail term. The subleading contribution also involves the phase correction, which causes the tail term to vanish asymptotically in the case of either ultrarelativistic or massless particles.

Several approaches to perturbations on the ReissnerNordström (RN) spacetime are known in the literature, notably those in [73-75]. However, our analysis in this paper will concern the weak field, fast-motion approximation which cannot be addressed using either the quadrupole moment formalism or the slow-motion approximation. The expansion in terms of generalized spherical harmonics is useful when the radiation is known to arise from specific multipole moments. The low frequency result then follows from taking the limit of the sum over moments, which in general is a more cumbersome route to soft limit expressions. For these reasons, in this paper we extend the result of $[2,4]$ and consider the scattering of a point particle with mass and charge on the four-dimensional RN spacetime, with the particle velocity taking on asymptotically large values. The scattering is considered in the probe limit with the gravitational and electromagnetic radiation results derived up to order $\frac{M}{r}$ and $\frac{Q}{r}$, where $M$ and $Q$ denote the mass and charge of the RN black hole. To the best of our knowledge, these classical solutions representing the gravitational and electromagnetic bremsstrahlung on the RN spacetime have not been previously derived in the literature.

We subsequently use our classical radiation results to demonstrate that they provide the expected tree-level soft factor involved in the soft photon and graviton theorems, including phases. The property of soft factors involving an independent sum over incoming and outgoing particles implies that we can use the universal contributions to investigate classical observables, in broader settings involving interacting masses and charges. We use the soft factors to determine the memory effect and its tail contribution in late time waveforms, resulting from scattering processes involving several outgoing light particles and no incoming light particles. Our results demonstrate that the charge contribution in the tail generically dominates the mass.
In considering the ultrarelativistic limit, both gravitational and electromagnetic wave tails vanish, consistent with the prior literature.

Our paper is organized as follows. In the following section, we first review perturbations of charged black hole spacetimes before arriving at the equations on the linearized RN spacetime. The pertubative solutions in frequency space are then derived using the scalar Green's function based on the worldline formalism on curved backgrounds. The derivation of the scalar Green's function on linearized curved backgrounds has been provided in Appendix A and specific expressions on the linearized RN spacetime have been derived in Appendix B. In Sec. III, we use the classical radiative solutions to determine the photon and graviton soft factors. We first review the results of $[47,70,71]$, which cover the predicted soft factors, the phase corrections involved, and the integral identities needed to derive these expressions from classical radiative solutions. We then derive the photon soft factor in Sec. III B and the graviton soft factor in Sec. III C, with technical details concerning the evaluation of the integrals provided in Appendix C. Following [72], in Sec. III D we determine the memory effect and its tail contribution in the late time gravitational and electromagnetic waveforms using the soft factor expressions. We conclude with a summary of our results.

\section{CLASSICAL RADIATIVE SOLUTIONS OF PROBE SCATTERING ON THE RN SPACETIME}

In this section, we will derive the metric and electromagnetic perturbations of the $\mathrm{RN}$ spacetime due to the scattering of a massive and charged point particle traveling on an unbound trajectory with a large impact parameter from the central black hole. We will follow the procedure used to calculate metric perturbations resulting from a point mass [4] and electromagnetic perturbations due to a point charge [2] on the Schwarzschild spacetime. In the following subsection, we consider linear perturbations of the Einstein-Maxwell equations of charged black hole spacetimes due to a point particle with mass and charge. We then specifically consider these perturbations on the RN spacetime up to linear order in $\frac{M}{r}$ and $\frac{Q}{r}$, in accordance with our assumption of a large impact parameter. The perturbations are then solved in the last subsection in frequency space by using the worldline formalism for the scalar Green's function.

\section{A. Perturbations of Einstein-Maxwell equations}

We consider the background to be a charged black hole spacetime which is a solution of the Einstein-Maxwell action. The solution satisfies Einstein's equations

$$
G_{\mu \nu}-T_{\mu \nu}=0
$$

and the source-free Maxwell's equations 


$$
F^{\mu \nu} ;_{\nu}=0
$$

where

$$
\begin{aligned}
G_{\mu \nu} & =R_{\mu \nu}-\frac{R}{2} g_{\mu \nu}, \quad F_{\mu \nu}=A_{\nu, \mu}-A_{\mu, \nu}, \\
T_{\mu \nu} & =\frac{1}{4 \pi}\left(F_{\mu \alpha} F_{\nu \beta} g^{\alpha \beta}-\frac{1}{4} g_{\mu \nu} F_{\alpha \beta} F_{\gamma \delta} g^{\alpha \gamma} g^{\beta \delta}\right) .
\end{aligned}
$$

The background metric and gauge potential are $g_{\mu \nu}$ and $A_{\mu}$. We adopt the mostly plus convention for the metric and set $8 \pi G=1$. Semicolons indicate covariant derivatives, while commas denote partial derivatives. Our convention for the Riemann tensor is

$$
R_{\mu \beta \nu}^{\alpha}=\Gamma_{\mu \nu, \beta}^{\alpha}-\Gamma_{\mu \beta}^{\alpha}, \nu+\Gamma_{\beta \gamma}^{\alpha} \Gamma_{\mu \nu}^{\gamma}-\Gamma_{\nu \gamma}^{\alpha} \Gamma_{\mu \beta}^{\gamma},
$$

where

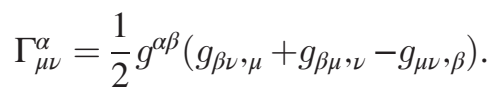

We now linearly perturb the spacetime by introducing a point particle with mass and charge. The corresponding action is

$$
S_{P}=-m \int d \sigma \sqrt{-g_{\mu \nu} \frac{d r^{\mu}}{d \sigma} \frac{d r^{\nu}}{d \sigma}}+\frac{q}{4 \pi} \int d \sigma A_{\mu} \frac{d r^{\mu}}{d \sigma} .
$$

From the variation of this action, we derive the following stress tensor $T_{(P)}^{\mu \nu}$ and current $J_{(P)}^{\mu}$

$$
\begin{aligned}
T_{(P)}^{\mu \nu} & =m \int \delta(x, r(\sigma)) \frac{d r^{\mu}}{d \sigma} \frac{d r^{\nu}}{d \sigma} d \sigma, \\
J_{(P)}^{\mu} & =\frac{q}{4 \pi} \int \delta(x, r(\sigma)) \frac{d r^{\mu}}{d \sigma} d \sigma
\end{aligned}
$$

where $\delta(x, r(\sigma))$ refers to the covariant delta function which is normalized as

$$
\int \sqrt{-g} \delta(x, r(\sigma)) d \sigma=1
$$

The stress-energy tensor and current of the point particle induces a perturbation of the metric and gauge potential which we denote by $h_{\mu \nu}$ and $a_{\mu}$ respectively, i.e.,

$$
\begin{aligned}
g_{\mu \nu} & \rightarrow g_{\mu \nu}+\delta g_{\mu \nu}=g_{\mu \nu}+2 h_{\mu \nu}, \\
A_{\mu} & \rightarrow A_{\mu}+\delta A_{\mu}=A_{\mu}+a_{\mu} .
\end{aligned}
$$

The variation of Eq. (2.1) and Eq. (2.2) provides the following equations:

$$
\begin{gathered}
\delta G_{\mu \nu}-\delta T_{\mu \nu}^{h}-\delta T_{\mu \nu}^{a}=T_{\mu \nu}^{(P)} \\
\delta\left(F^{\mu \nu}{ }_{\nu}\right)=4 \pi J_{(P)}^{\mu},
\end{gathered}
$$

where we have denoted the perturbation of the stressenergy tensor as a combination of terms involving perturbations of the metric $\delta T_{\mu \nu}^{h}$ and perturbations of the gauge potential $\delta T_{\mu \nu}^{a}$ in Eq. (2.9).

We will denote the perturbed electromagnetic field strength tensor by

$$
f_{\mu \nu}=a_{\nu, \mu}-a_{\mu, \nu} .
$$

It will also be convenient to describe the perturbation equations in terms of trace-reversed metric perturbations $e_{\mu \nu}$ defined by

$$
e_{\mu \nu}=h_{\mu \nu}-\frac{1}{2} h g_{\mu \nu} ; \quad h=g^{\mu \nu} h_{\mu \nu}=-g^{\mu \nu} e_{\mu \nu}=-e .
$$

The simplified expressions for $\delta G_{\mu \nu}, \delta T_{\mu \nu}^{h}, \delta T_{\mu \nu}^{a}$ and $\delta\left(F^{\mu \nu} ;_{\nu}\right)$ in terms of $e_{\mu \nu}$ and $f_{\mu \nu}$ are

$$
\begin{aligned}
\delta G_{\mu \nu}= & -e_{\mu \nu ; \alpha}{ }^{\alpha}+e_{\mu \alpha ;}{ }^{\alpha}{ }_{\nu}+e_{\nu \alpha ;}{ }^{\alpha}{ }_{\mu}+\left(R_{\nu}{ }^{\delta} e_{\delta \mu}+R_{\mu}{ }^{\delta} e_{\delta \nu}\right)+2 R_{\nu \mu}^{\alpha}{ }_{\nu \mu} e_{\delta \alpha} \\
& -g_{\mu \nu} e_{\alpha \beta ;}{ }^{\alpha \beta}-R e_{\mu \nu}+g_{\mu \nu} R^{\alpha \beta} e_{\alpha \beta} \\
\delta T_{\mu \nu}^{h}= & e T_{\mu \nu}-\frac{1}{8 \pi} e_{\mu \nu} F_{\alpha \beta} F^{\alpha \beta}-\frac{1}{2 \pi} g^{\alpha \epsilon} g^{\beta \delta} e_{\epsilon \delta}\left(F_{\alpha \mu} F_{\beta \nu}-\frac{1}{4} g_{\mu \nu} F_{\alpha \gamma} F_{\beta}^{\gamma}\right) \\
\delta T_{\mu \nu}^{a}= & \frac{1}{4 \pi} g^{\alpha \beta}\left(f_{\alpha \mu} F_{\beta \nu}+f_{\alpha \nu} F_{\beta \mu}-\frac{1}{2} g_{\mu \nu} g^{\gamma \delta} f_{\alpha \gamma} F_{\beta \delta}\right) \\
\delta\left(F^{\mu \nu} ;_{\nu}\right)= & -g^{\alpha \rho} g^{\mu \nu}\left(2 g^{\beta \sigma} e_{\rho \sigma} F_{\nu \beta} ;_{\alpha}+2 g^{\beta \sigma} e_{\nu \rho ; \sigma} F_{\alpha \beta}-e_{; \rho} F_{\nu \alpha}-f_{\nu \rho} ;_{\alpha}-2 F_{\alpha \nu} e_{\rho \beta} ;^{\beta}\right) .
\end{aligned}
$$

By substituting the first three expressions of Eq. (2.13) in Eq. (2.9), we find the following expression for the perturbed Einstein equation: 


$$
\begin{aligned}
-T_{\mu \nu}^{(P)}= & e_{\mu \nu ; \alpha}{ }^{\alpha}-e_{\mu \alpha ;}{ }_{\nu}-e_{\nu \alpha ; \mu}^{\alpha}-\left(R_{\nu}{ }^{\delta} e_{\delta \mu}+R_{\mu}{ }^{\delta} e_{\delta \nu}\right)-2{R^{\alpha \mu}}_{\nu \mu}{ }^{\delta} e_{\delta \alpha}+R e_{\mu \nu} \\
& -g_{\mu \nu} R^{\alpha \beta} e_{\alpha \beta}+g_{\mu \nu} e_{\alpha \beta}{ }^{\alpha \beta}+\frac{1}{4 \pi} g^{\alpha \beta}\left(f_{\alpha \mu} F_{\beta \nu}+f_{\alpha \nu} F_{\beta \mu}-\frac{1}{2} g_{\mu \nu}{ }^{\gamma \delta} f_{\alpha \gamma} F_{\beta \delta}\right) \\
& +e T_{\mu \nu}-\frac{1}{8 \pi} e_{\mu \nu} F_{\alpha \beta} F^{\alpha \beta}-\frac{1}{2 \pi} g^{\alpha \epsilon} g^{\beta \delta} e_{\epsilon \delta}\left(F_{\alpha \mu} F_{\beta \nu}-\frac{1}{4} g_{\mu \nu} F_{\alpha \gamma} F_{\beta}^{\gamma}\right),
\end{aligned}
$$

while using the last line of Eq. (2.13) in Eq. (2.10) gives us the perturbed Maxwell equation

$$
-4 \pi g^{\mu \nu} J_{\nu}^{(P)}=g^{\alpha \rho} g^{\mu \nu}\left(2 g^{\beta \sigma} e_{\rho \sigma} F_{\nu \beta} ;_{\alpha}+2 g^{\beta \sigma} e_{\nu \rho ; \sigma} F_{\alpha \beta}-e_{; \rho} F_{\nu \alpha}-f_{\nu \rho} ;_{\alpha}-2 F_{\alpha \nu} e_{\rho \beta}^{\beta}{ }^{\beta}\right) .
$$

In general, the conservation equation requires

$$
\delta G_{\mu \nu}{ }^{\mu}-\delta T_{\mu \nu ;}^{h}{ }^{\mu}-\delta T_{\mu \nu ;}^{a}{ }^{\mu}-T_{\mu \nu ;}^{(P) \mu}=0
$$

\section{B. Perturbations on the linearized RN spacetime}

We will now consider the above perturbations on the $\mathrm{RN}$ spacetime. In isotropic coordinates, the metric is given by

$$
d s^{2}=-g_{00} d t^{2}+g_{i j} d x^{i} d x^{j}
$$

with

$$
\begin{aligned}
& g_{00}=-\left(\frac{1-\frac{1}{4 R^{2}}\left(\frac{M^{2}}{64 \pi^{2}}-\frac{Q^{2}}{8 \pi}\right)}{\left(1+\frac{M}{16 \pi R}\right)^{2}-\frac{1}{8 \pi}\left(\frac{Q}{2 R}\right)^{2}}\right)^{2}, \\
& g_{i j}=\delta_{i j}\left(\left(1+\frac{M}{16 \pi R}\right)^{2}-\frac{1}{8 \pi}\left(\frac{Q}{2 R}\right)^{2}\right)^{2},
\end{aligned}
$$

where $R=|\vec{x}|$. The parameters $M$ and $Q$ refer to the mass and charge, respectively, of the RN black hole. The gauge field $A_{\mu}$ has the following nonvanishing component:

$$
A_{0}=\frac{Q}{R}\left(1+\frac{M}{8 \pi R}+\frac{1}{4 R^{2}}\left(\frac{M^{2}}{64 \pi^{2}}-\frac{Q^{2}}{8 \pi}\right)\right)^{-1}
$$

Equations (2.17) and (2.19) satisfy the Einstein-Maxwell equations in Eq. (2.1) and Eq. (2.2). In considering perturbations about this background along the lines of the previous section, we will implement a few approximations. First, we will be working in the probe scattering limit, where the point particle is assumed to have a large impact parameter from the central black hole. This amounts to the assumption that we consider $\frac{M}{R} \ll 1$ and $\frac{Q}{R} \ll 1$. Second, the mass $m$ and charge $q$ of the point particle are considered to be much smaller than the mass $M$ and charge $Q$ of the RN black hole. Specifically, we make the assumption that $M \gg m$ and $Q \gg q$. These assumptions allow us to consider our perturbations about the linearized RN spacetime, where the metric and gauge potential will be considered only up to terms which are linear in $Q$ and $M$.
We will express the metric and gauge field in terms of the potential

$$
\phi(\vec{x})=-\frac{M}{8 \pi R}
$$

Hence all $\mathcal{O}\left(\phi^{2}\right)$ contributions will contain the $\mathcal{O}\left(M^{2}\right)$, $\mathcal{O}(Q M)$, and $\mathcal{O}\left(Q^{2}\right)$ terms. Ignoring $\mathcal{O}\left(\phi^{2}\right)$ corrections, the metric components then have the form

$g_{00}=-(1+2 \phi), \quad g_{0 i}=0, \quad g_{i j}=\delta_{i j}(1-2 \phi)$,

while the gauge potential in this approximation is

$$
A_{0}(\vec{x})=-\frac{8 \pi Q}{M} \phi(\vec{x}) .
$$

We will denote $\frac{\partial}{\partial x^{i}}$ as $\partial_{i}$ and $\frac{\partial}{\partial x^{0}}$ as $\partial_{0}$. We then have from Eq. (2.21) the following nonvanishing connection, Riemann and Ricci tensor components

$$
\begin{aligned}
\Gamma_{0 i}^{0} & =\phi_{, i}=\Gamma_{00}^{i}, \quad \Gamma_{j k}^{i}=\delta_{j k} \phi_{, i}-\delta_{i j} \phi_{, k}-\delta_{i k} \phi_{, j}, \\
R^{0}{ }_{i j 0} & =\phi_{, i j}, \quad R^{k}{ }_{i j m}=\delta_{i m} \phi_{, k j}+\delta_{j k} \phi_{, i m}-\delta_{i j} \phi_{, m k}-\delta_{m k} \phi_{, i j} \\
R_{00} & =\phi_{, k k}, \quad R_{i j}=\delta_{i j} \phi_{, k k}, \\
R & =2 \phi_{, k k}
\end{aligned}
$$

while Eq. (2.22) gives us the nonvanishing electromagnetic field strength tensor component

$$
F_{0 i}=\frac{8 \pi Q}{M} \phi_{, i}
$$

At the linearized level, the stress-energy tensor of the Maxwell field goes like $\mathcal{O}\left(\phi^{2}\right)$. Indeed, this also implies that the linearized RN spacetime appears to be exactly as that of linearized Schwarzschild spacetime with a gauge potential. More significantly, however, perturbations about the linearized RN spacetime are different from those about the linearized Schwarzschild spacetime, which will be relevant in the following analysis. Thus while $\delta T_{\mu \nu}^{h}$ 
vanishes at the linearized level, contributions from $\delta T_{\mu \nu}^{a}$ are present in the perturbed Einstein equation in Eq. (2.14). Likewise, the perturbed Maxwell equation in Eq. (2.15) involves $e_{\mu \nu}$ corrections unlike the perturbations about the Schwarzschild spacetime.

We will now substitute Eqs. (2.17)-(2.24) in Eqs. (2.14) and (2.15). Let us define

$$
\square=\eta^{\mu \nu} \partial_{\mu} \partial_{\nu}=-\partial_{0}^{2}+\partial_{i}^{2}
$$

and

$$
k_{\mu}=e_{\mu \nu} ; \nu, \quad l=-a_{0,0}+a_{i, i} .
$$

We will be interested in the radiative components of the gravitational and electromagnetic fields. These components will only require us to consider the spatial components of the perturbed fields. As we will note, other components of the perturbed fields can be determined from the gauge fixing choices we will adopt. For the $i j$ component of the perturbed Einstein equation, Eq. (2.14), we find the following expression:

$$
\begin{aligned}
-T_{i j}^{(P)}= & \square\left((1+2 \phi) e_{i j}\right)-k_{i, j}-k_{j, i}-2\left(\phi_{, k} e_{k i}\right)_{, j}-2\left(\phi_{, k} e_{k j}\right)_{, i}-\left(k_{0,0}-k_{l, l}\right) \delta_{i j} \\
& +4\left[\phi e_{i j, 00}+\phi_{, i} e_{0 j, 0}+\phi_{, j} e_{i 0,0}+\frac{1}{2}\left(\phi_{, i j}-\frac{1}{2} \phi_{k k} \delta_{i j}\right)\left(e_{00}+e_{l l}\right)\right] \\
& +2 \delta_{i j}\left(\phi_{, k l} e_{k l}-\phi_{, k} k_{k}+2 \phi k_{0,0}\right)-\frac{2 Q}{M}\left(f_{0 i} \phi_{, j}+f_{0 j} \phi_{, i}-\delta_{i j} f_{0 l} \phi_{, l}\right),
\end{aligned}
$$

while the $i$ component of the perturbed Maxwell equation, Eq. (2.15), gives us

$$
\begin{aligned}
-(1+2 \phi) 4 \pi J_{i}^{(P)}= & \square a_{i}-l_{, i}+2 \phi_{, k} f_{k i}+4 \phi\left(a_{i, k k}-a_{k, k i}\right)+\frac{16 \pi Q}{M}\left(e_{0 j} \phi_{, i j}+\phi_{, i} k_{0}\right. \\
& \left.+\left(e_{i j, 0}-e_{i 0, j}\right) \phi_{, j}+\frac{1}{2}\left(e_{00,0}-e_{k k, 0}\right) \phi_{, i}\right) .
\end{aligned}
$$

We now need to fix a gauge in Eqs. (2.27) and (2.28). It is desirable that any choice we make for $k_{\mu}$ and $l$ reduce to the usual flat spacetime de Donder and Lorenz gauges in the $\phi \rightarrow 0$ limit. However, the key principle in our choice will be to ensure that all mixed terms with $\phi$ involve only time derivatives of the perturbed fields. This will be crucial for solving the perturbations using the approach described in the next subsection.

Thus in Eq. (2.27), we need to implement a choice for $k_{\mu}$ such that it eliminates the terms

$$
-2\left(\left(\phi_{, k} e_{k i}\right)_{, j}+\left(\phi_{, k} e_{k j}\right)_{, i}-\delta_{i j} \phi_{, k l} e_{k l}\right)+\frac{2 Q}{M}\left(a_{0, i} \phi_{, j}+a_{0, j} \phi_{, i}-\delta_{i j} a_{0, l} \phi_{, l}\right) .
$$

We note that the following gauge choice for $k_{\mu}$ achieves this:

$$
k_{\mu}=-2 \phi_{, k} e_{k \mu}+\frac{2 Q}{M} a_{0} \phi_{, \mu}
$$

Substituting this expression for $k_{\mu}$ in Eq. (2.27) gives us the desired gauge fixed expression

$$
\begin{aligned}
-T_{i j}^{(P)}= & \square\left((1+2 \phi) e_{i j}\right)+4\left[\phi e_{i j, 00}+\phi_{, i} e_{0 j, 0}+\phi_{, j} e_{i 0,0}+\frac{1}{2}\left(\phi_{, i j}-\frac{1}{2} \phi_{k k} \delta_{i j}\right)\left(e_{00}+e_{l l}\right)\right] \\
& -\frac{2 Q}{M}\left[a_{i, 0} \phi_{, j}+a_{j, 0} \phi_{, i}-\delta_{i j} a_{l, 0} \phi_{, l}+2 a_{0}\left(\phi_{, i j}-\frac{1}{2} \delta_{i j} \phi_{k k}\right)\right] .
\end{aligned}
$$

Substituting Eq. (2.30) in Eq. (2.28) also eliminates the $k_{\mu}$ contribution in the electromagnetic perturbation equation, since these contributions are $\mathcal{O}\left(\phi^{2}\right)$. The terms involving spatial derivatives of $a_{i}$ in the electromagnetic pertubation equation are

$$
2 \phi_{, k} f_{k i}+4 \phi\left(a_{i, k k}-a_{k, k i}\right),
$$

which can be addressed with the following choice for $l$ : 


$$
l=-4 \phi a_{k, k}-2 \phi_{, k} a_{k} .
$$

We now substitute Eq. (2.33) in Eq. (2.28) to find the gauge fixed expression

$$
\begin{aligned}
-(1-\phi) 4 \pi J_{i}^{(P)}= & \square\left((1+\phi) a_{i}\right)+4\left[\phi a_{i, 00}+\phi_{, i} a_{0,0}\right]-\phi_{, k k} a_{i}+2 \phi_{, i k} a_{k} \\
& +\frac{16 \pi Q}{M}\left(e_{0 j} \phi_{, i j}+\left(e_{i j, 0}-e_{i 0, j}\right) \phi_{, j}+\frac{1}{2}\left(e_{00,0}-e_{k k, 0}\right) \phi_{, i}\right),
\end{aligned}
$$

where we made use of the following property resulting from Eq. (2.33):

$$
\phi l=0=\phi\left(-a_{0,0}+a_{k, k}\right) .
$$

In the following subsections, we will proceed to solve the equations for $e_{i j}$ and $a_{i}$ given in Eqs. (2.31) and (2.34), respectively. These solutions will be determined in frequency space following a Fourier transform in time. The procedure which will be used to derive the solutions is the worldline formalism applied to the scalar Green's function.

\section{Solutions using the worldline formalism}

Equations (2.31) and (2.34) can be viewed as differential equations involving the flat spacetime D'Alembertian operator and subleading terms involving $\phi$ and its derivatives. To obtain the solutions of such equations in frequency space we will need the solution of the following equation:

$$
\psi_{; \alpha}^{(1) \alpha}=-\int \delta(x, r(\sigma)) f(\sigma) d \sigma+\mathcal{O}\left(R^{2}\right),
$$

with the form of $f(\sigma)$ depending on the source and where the covariant delta function $\delta(x, r(\sigma))$ is related to the flat spacetime delta function $\delta^{4}(x-r(\sigma))$ via

$\delta(x, r(\sigma)) \sqrt{-g}=\delta^{4}(x-r(\sigma))=\delta\left(t-r^{0}(\sigma)\right) \delta^{(3)}(\vec{x}-\vec{r}(\sigma))$.

The solution of Eq. (2.36) which can be derived using the worldline formalism [76,77] is [78]

$$
\psi^{(1)}=\psi^{(0)}+\delta \psi^{(0)},
$$

where

$$
\begin{aligned}
\psi^{(0)}(x)= & \frac{1}{4 \pi} \int_{-\infty}^{\sigma_{0}} \delta(-\Omega(x, r(\sigma))) f(\sigma) d \sigma, \\
\delta \psi^{(0)}(x)= & \frac{1}{16 \pi^{2}} \int \sqrt{-g(y)} \delta(-\Omega(x, y)) d^{4} y \\
& \times \int_{-\infty}^{\sigma_{0}} \delta^{\prime}(-\Omega(y, r(\sigma))) F(y, r(\sigma)) f(\sigma) d \sigma .
\end{aligned}
$$

In Eq. (2.39), the world function $\Omega(x, r(\sigma))$ is defined in terms of the geodesic $U^{\mu}$ which connects the point $x$ at parametric value $u_{1}$ and $r(\sigma)$ at parametric value $u_{0}$

$$
\Omega(x, r(\sigma))=\frac{u_{1}-u_{0}}{2} \int_{u_{0}}^{u_{1}} g_{\mu \nu} U^{\mu} U^{\nu} d u,
$$

while $F(y, r(\sigma))$ in Eq. (2.40) is further defined using the Ricci tensor $R_{\mu \nu}$

$F(y, r(\sigma))=\frac{1}{u_{1}-u_{0}} \int_{u_{0}}^{u_{1}}\left(u-u_{0}\right)^{2} R_{\mu \nu} U^{\mu} U^{\nu} d u$.

We note at this point that while $\psi^{(0)}$ does involve terms that are both independent and linear in curvature, $\delta \psi^{(0)}(x)$ only involves curvature dependent terms. Let us now consider the specific case of the linearized RN spacetime, whose metric is given in Eq. (2.21). By expanding Eq. (2.36) explicitly in terms of $\phi$, we find

$$
\square \psi^{(1)}+4 \phi \partial_{t}^{2} \psi^{(1)}=-\int \delta^{4}(x-r(\sigma)) f(\sigma) d \sigma+\mathcal{O}\left(R^{2}\right) .
$$

The source term in Eq. (2.43) is now simply that for Green's function in flat spacetime. This suggests that we can assume $\psi^{(0)}$ to be composed in the following way:

$$
\psi^{(0)}=\psi_{0}^{(0)}+\psi_{1}^{(0)}
$$

where $\psi_{0}^{(0)}$ manifestly satisfies the flat spacetime equation for Green's function, while $\psi_{1}^{(0)}$ is an additional contribution which does not. From Eq. (2.43), we then find the following two equalities:

$$
\begin{gathered}
\square \psi_{0}^{(0)}=-\int \delta^{4}(x-r(\sigma)) f(\sigma) d \sigma, \\
-4 \phi \partial_{t}^{2} \psi^{(0)}=\square\left(\psi_{1}^{(0)}+\delta \psi^{(0)}\right) .
\end{gathered}
$$

Equations (2.45) and (2.46) further imply that in considering perturbative solutions about the linearized RN spacetime, we can simply express Eq. (2.43) as 


$$
\begin{aligned}
& \square\left(\psi_{0}^{(0)}-\psi_{1}^{(0)}-\delta \psi^{(0)}\right) \\
& \quad=-\int \delta^{4}(x-r(\sigma)) f(\sigma) d \sigma+\mathcal{O}\left(R^{2}\right) .
\end{aligned}
$$

Hence on the linearized RN spacetime, we can express Eq. (2.43) as an equation for the scalar Green's function on flat spacetime. Furthermore, we can also derive solutions when we consider specific corrections of Eq. (2.43), as in the case of Eq. (2.31) and Eq. (2.34). Should Eq. (2.43) contain correction terms involving derivatives of $\phi$ and time derivatives of $\psi^{(1)}$, then Eq. (2.46) can be used to find an expression involving the flat spacetime D'Alembertian operator acting on the additional terms. We may thus perturbatively construct solutions in these cases as well.

In Appendix B, we have explicitly derived the following solutions for $\psi^{(0)}$ and $\delta \psi^{(0)}$ on the linearized RN spacetime:

$$
\begin{aligned}
\psi^{(0)}(t, \vec{x})= & \frac{1}{4 \pi} \int_{-\infty}^{\infty} \frac{\delta\left(t-r^{0}-R_{0}-\frac{M}{4 \pi} \Gamma(\vec{x}, \vec{z})\right)}{R_{0}} f(\sigma) d \sigma, \\
\delta \psi^{(0)}(t, \vec{x})= & \frac{M}{16 \pi^{2}} \partial_{t} \int_{0}^{\infty} d v \\
& \times \int_{-\infty}^{\infty} d \sigma \frac{\delta\left(t-r^{0}-|\vec{r}|-v-\rho(v)\right)}{\rho(v)(|\vec{r}|+v)} f(\sigma),
\end{aligned}
$$

where as before $\vec{R}_{0}=\vec{x}-\vec{r}, R=|\vec{x}|$ and

$\Gamma(\vec{x}, \vec{r})=\ln \left(\frac{R R_{0}+\vec{x} \cdot \vec{r}}{|\vec{r}| R_{0}+\vec{z} \cdot \vec{r}}\right), \quad \rho(v)=\sqrt{R^{2}+v^{2}+\frac{2 v \vec{x} . \vec{r}}{|\vec{r}|}}$

The solutions in Eqs. (2.48) and (2.49) are the same as those about the linearized Schwarzschild spacetime [77].

To derive the perturbative solutions in frequency space, we perform the Fourier transform

$$
\begin{aligned}
\tilde{\psi}(\omega, \vec{x}) & =\frac{1}{2 \pi} \int d t e^{i \omega t} \psi(t, \vec{x}), \\
\psi(t, \vec{x}) & =\int d \omega e^{-i \omega t} \tilde{\psi}(\omega, \vec{x}),
\end{aligned}
$$

where $\psi$ refers to any of the fields mentioned above $\psi^{(1)}, \psi_{0}^{(0)}, \psi_{1}^{(0)}$, or $\delta \psi^{(0)}$. By Fourier transforming Eqs. (2.48) and (2.49) [using Eq. (2.51)], we find

$$
\begin{aligned}
\tilde{\psi}^{(0)}(\omega, \vec{x})= & \frac{1}{4 \pi} \int_{-\infty}^{\infty} \frac{e^{i \omega\left(r^{0}+R_{0}\right)}}{R_{0}} f(\sigma) d \sigma \\
& +\frac{i \omega M}{16 \pi^{2}} \int_{-\infty}^{\infty} \frac{e^{i \omega\left(r^{0}+R_{0}\right)} \Gamma(\vec{x}, \vec{r})}{R_{0}} f(\sigma) d \sigma, \quad(2.53) \\
\delta \tilde{\psi}^{(0)}(\omega, \vec{x})= & -\frac{i \omega M}{16 \pi^{2}} \int_{0}^{\infty} d v \int_{-\infty}^{\infty} d \sigma \frac{e^{i \omega\left(r^{0}+v+|\vec{r}|+\rho(v)\right)}}{(v+|\vec{r}|) \rho(v)} f(\sigma) .
\end{aligned}
$$

From Eq. (2.53), we further identify

$$
\tilde{\psi}_{0}^{(0)}(\omega, \vec{x})=\frac{1}{4 \pi} \int_{-\infty}^{\infty} \frac{e^{i \omega\left(r^{0}+R_{0}\right)}}{R_{0}} f(\sigma) d \sigma
$$

$\tilde{\psi}_{1}^{(0)}(\omega, \vec{x})=\frac{i \omega M}{16 \pi^{2}} \int_{-\infty}^{\infty} \frac{e^{i \omega\left(r^{0}+R_{0}\right)} \Gamma(\vec{x}, \vec{r})}{R_{0}} f(\sigma) d \sigma$.

On substituting Eq. (2.52) in Eq. (2.45) and defining $\tilde{\square}:=\omega^{2}+\partial_{i}^{2}$, we find

$$
\int d \omega e^{-i \omega t} \tilde{\square} \tilde{\psi}_{0}^{(0)}(\vec{x}, \omega)=-\int \delta\left(t-r^{0}\right) \delta^{(3)}(\vec{x}-\vec{r}) f(\sigma) d \sigma,
$$

which is satisfied by the solution in Eq. (2.55). By Fourier transforming Eq. (2.46) and on using Eqs. (2.56) and (2.54) we find

$$
-\tilde{\square} G_{M}(\omega, \vec{x}, \vec{r})=-\left(\omega^{2}+\partial_{i}^{2}\right) G_{M}(\omega, \vec{x}, \vec{r})=\phi(\vec{x}) \frac{e^{i \omega R_{0}}}{R_{0}},
$$

where we have defined $G_{M}$ as

$$
\begin{aligned}
& G_{M}(\omega, \vec{x}, \vec{r}) \\
& \quad=-\frac{i M}{16 \pi \omega}\left(\frac{e^{i \omega R_{0}} \Gamma(\vec{x}, \vec{r})}{R_{0}}-\int_{0}^{\infty} d v \frac{e^{i \omega(v+|\vec{r}|+\rho(v))}}{(v+|\vec{r}|) \rho(v)}\right) .
\end{aligned}
$$

Equation (2.58) enables us to express $\phi$ and its derivatives in terms of $\tilde{\square} G_{M}$. Let us define the derivative $\nabla_{i}:=$ $\frac{\partial}{\partial x^{i}}+\frac{\partial}{\partial r^{r}}$. Since $\vec{R}_{0}=\vec{x}-\vec{r}$ we have $\nabla_{i} f\left(R_{0}\right)=0$, for $f\left(R_{0}\right)$ being some function of $R_{0}$. Hence we can conveniently describe derivatives of $\phi$ in the following way:

$$
\begin{aligned}
-\tilde{\square} \nabla_{i} G_{M}(\omega, \vec{x}, \vec{r}) & =\phi_{, i} \frac{e^{i \omega R_{0}}}{R_{0}}, \\
-\tilde{\square} \nabla_{i} \nabla_{k} G_{M}(\omega, \vec{x}, \vec{r}) & =\phi_{, i k} \frac{e^{i \omega R_{0}}}{R_{0}}, \quad \text { etc. }
\end{aligned}
$$




\section{Solutions of the perturbation equations}

Using the results of the previous subsection, we can now derive the solutions of Eqs. (2.31) and (2.34). These equations can be viewed as involving corrections of the equation for the scalar Green's function. In the case of Eq. (2.31), we have

$$
-T_{i j}^{(P)}=-m \int \delta_{k i} \delta_{l j} \frac{\delta^{4}(x-r(\sigma))}{1+2 \phi(\vec{r})} \frac{d r^{k}}{d \sigma} \frac{d r^{l}}{d \sigma} d \sigma=\square\left((1+2 \phi) e_{i j}\right)+4 \phi e_{i j, 00}+\cdots
$$

while for Eq. (2.34) we have

$$
-(1-\phi) 4 \pi J_{i}^{(P)}=-q \int \delta_{k i} \frac{\delta^{4}(x-r(\sigma))}{1+\phi(\vec{r})} \frac{d r^{k}}{d \sigma} d \sigma=\square\left((1+\phi) a_{i}\right)+4 \phi a_{i, 00}+\cdots
$$

where $\cdots$ in the right-hand side of Eqs. (2.61) and (2.62) denote the additional terms in the right-hand side of Eqs. (2.31) and (2.34), respectively. We see that Eqs. (2.61) and (2.62) are simply of the form of Eq. (2.43). Using Eq. (2.52) we Fourier transform the independent perturbed fields $e_{i j}$ and $a_{i}$

$$
e_{i j}(t, \vec{x})=\int d \omega e^{-i \omega t} \tilde{e}_{i j}(\omega, \vec{x}), \quad a_{i}(t, \vec{x})=\int d \omega e^{-i \omega t} \tilde{a}_{i}(\omega, \vec{x}) .
$$

Substituting Eq. (2.63) in Eq. (2.31) gives us

$$
\begin{aligned}
-T_{i j}^{(P)}= & -m \int \delta_{k i} \delta_{l j} \frac{\delta^{4}(x-r(\sigma))}{1+2 \phi(\vec{r})} \frac{d r^{k}}{d \sigma} \frac{d r^{l}}{d \sigma} d \sigma=\int d \omega e^{-i \omega t} \tilde{\square}\left((1+2 \phi) \tilde{e}_{i j}\right) \\
& -\int d \omega e^{-i \omega t} 4\left[\omega^{2} \phi \tilde{e}_{i j}+i \omega\left(\phi_{, i} \tilde{e}_{j 0}+\phi_{, j} \tilde{e}_{i 0}\right)-\frac{1}{2}\left(\phi_{, i j}-\frac{1}{2} \phi_{, k k} \delta_{i j}\right)\left(\tilde{e}_{00}+\tilde{e}_{l l}\right)\right] \\
& +\frac{2 Q}{M} \int d \omega e^{-i \omega t}\left[i \omega\left(\tilde{a}_{i} \phi_{, j}+\tilde{a}_{j} \phi_{, i}-\delta_{i j} \tilde{a}_{l} \phi_{, l}\right)-2 \tilde{a}_{0}\left(\phi_{, i j}-\frac{1}{2} \delta_{i j} \phi_{, k k}\right)\right]
\end{aligned}
$$

and substituting Eq. (2.63) in Eq. (2.34) gives

$$
\begin{aligned}
-(1-\phi) 4 \pi J_{i}^{(P)}= & -q \int \delta_{k i} \frac{\delta^{4}(x-r(\sigma))}{1+\phi(\vec{r})} \frac{d r^{k}}{d \sigma} d \sigma=\int d \omega e^{-i \omega t} \tilde{\square}\left((1+\phi) \tilde{a}_{i}\right) \\
& -\int d \omega e^{-i \omega t}\left[4\left(\omega^{2} \tilde{a}_{i} \phi+i \omega \tilde{a}_{0} \phi_{, i}\right)+\tilde{a}_{i} \phi_{, k k}-2 \tilde{a}_{k} \phi_{, i k}\right] \\
& +\frac{16 \pi Q}{M} \int d \omega e^{-i \omega t}\left[\tilde{e}_{0 j} \phi_{, i j}-i \omega \tilde{e}_{i j} \phi_{, j}-\tilde{e}_{i 0, j} \phi_{, j}-\frac{1}{2} i \omega\left(\tilde{e}_{00}-\tilde{e}_{k k}\right) \phi_{, i}\right] .
\end{aligned}
$$

Comparing the first lines of Eqs. (2.64) and (2.65) with Eq. (2.57) allows us to determine the analog of the leading contribution $\tilde{\psi}_{0}^{(0)}$ in these equations, whose solution is given in Eq. (2.55). Choosing $\tilde{\psi}_{0}^{(0)}(\omega, \vec{x})=(1+2 \phi(\vec{x})) \times \tilde{e}_{i j}(\omega, \vec{x})$ and $f(\sigma)=\delta_{k i} \delta_{l j} \frac{m}{1+2 \phi(\vec{r})} \frac{d r^{k}}{d \sigma} \frac{d r^{l}}{d \sigma}$, we find on comparing with Eqs. (2.57) and (2.55) the following leading order contribution $\tilde{e}_{i j}^{(0)}(\omega, \vec{x})$ in the solution of Eq. (2.61) in frequency space:

$$
\tilde{e}_{i j}^{(0)}(\omega, \vec{x})=\frac{m}{1+2 \phi(\vec{x})} \int \frac{e^{i \omega\left(r^{0}+R_{0}\right)}}{4 \pi R_{0}} \frac{v_{i} v_{j}}{1+2 \phi(\vec{r})} \frac{d r^{0}}{d \sigma} d r^{0}
$$

where we changed the integration variable from $\sigma$ to $r^{0}$ in the final expression of the solution and have denoted $\frac{d r^{k}}{d r^{0}}$ as $v^{k}$. Likewise, on choosing $\tilde{\psi}_{0}^{(0)}(\omega, \vec{x})=(1+\phi(\vec{x})) \tilde{a}_{i}(\omega, \vec{x})$ and $f(\sigma)=\delta_{k i} \frac{q}{1+\phi(\vec{r})} \frac{d r^{k}}{d \sigma}$, we can compare with Eqs. (2.57) and (2.55) to find the following leading order contribution $\tilde{a}_{i}^{(0)}(\omega, \vec{x})$ in the solution of Eq. (2.62):

$$
\tilde{a}_{i}^{(0)}(\omega, \vec{x})=\frac{q}{1+\phi(\vec{x})} \int \frac{e^{i \omega\left(r^{0}+R_{0}\right)}}{4 \pi R_{0}} \frac{v_{i}}{1+\phi(\vec{r})} d r^{0}
$$


Equations (2.66) and (2.67) would be the complete solutions of Eqs. (2.64) and (2.65), respectively, in the absence of the second and third lines of Eqs. (2.64) and (2.65). The solution in the presence of these additional terms can be determined in the following way. First, since Eqs. (2.64) and (2.65) can be viewed as involving corrections for the equation for the scalar Green's function, we can make use of Eq. (2.58) for all terms in Eqs. (2.66) and (2.67) apart from those in the first line. Second, as these terms all involve $\phi$ or their derivatives, we can consider the gauge conditions in Eqs. (2.30) and (2.33) to lowest order in $\phi$. The resulting equations from the gravitational gauge condition in Eq. (2.30) are

$$
e_{i j, j}-e_{i 0,0}=0, \quad e_{0 i, i}-e_{00,0}=0,
$$

while the equation resulting from Eq. (2.33) is

$$
a_{0,0}-a_{i, i}=0 .
$$

These are simply the de Donder and Lorentz gauges in flat spacetime. By Fourier transforming Eq. (2.68) and using Eq. (2.66), we derive the following lowest order in $\phi$ solutions:

$$
\begin{aligned}
& \tilde{e}_{i j}^{(0)}(\omega, \vec{x})=m \int \frac{e^{i \omega\left(r^{0}+R_{0}\right)}}{4 \pi R_{0}} v_{i} v_{j} \frac{d r^{0}}{d \sigma} d r^{0}+\mathcal{O}(\phi), \\
& \tilde{e}_{i 0}^{(0)}(\omega, \vec{x})=-m \int \frac{e^{i \omega\left(r^{0}+R_{0}\right)}}{4 \pi R_{0}} v_{i} \frac{d r^{0}}{d \sigma} d r^{0}+\mathcal{O}(\phi), \\
& \tilde{e}_{00}^{(0)}(\omega, \vec{x})=m \int \frac{e^{i \omega\left(r^{0}+R_{0}\right)}}{4 \pi R_{0}} \frac{d r^{0}}{d \sigma} d r^{0}+\mathcal{O}(\phi) .
\end{aligned}
$$

Using Eq. (2.67) with the Fourier transform in Eq. (2.69) identifies the lowest order in $\phi$ solutions for electromagnetic perturbations

$$
\begin{aligned}
& \tilde{a}_{i}^{(0)}(\omega, \vec{x})=q \int \frac{e^{i \omega\left(r^{0}+R_{0}\right)}}{4 \pi R_{0}} v_{i} d r^{0}+\mathcal{O}(\phi), \\
& \tilde{a}_{0}^{(0)}(\omega, \vec{x})=-q \int \frac{e^{i \omega\left(r^{0}+R_{0}\right)}}{4 \pi R_{0}} d r^{0}+\mathcal{O}(\phi) .
\end{aligned}
$$

Hence the gauge conditions determine all the lowest order expressions. Substituting Eqs. (2.70) and (2.71) in Eqs. (2.64) and (2.65), we then use the expressions in Eqs. (2.58) and (2.60) to determine the following solutions for $\tilde{e}_{i j}(\omega, \vec{x})$ and $\tilde{a}_{i}(\omega, \vec{x})$ :

$$
\begin{aligned}
\tilde{e}_{i j}(\omega, \vec{x})= & \frac{m}{1+2 \phi(\vec{x})} \int \frac{e^{i \omega\left(r^{0}+R_{0}\right)}}{4 \pi R_{0}} \frac{v_{i} v_{j}}{1+2 \phi(\vec{r})} \frac{d r^{0}}{d \sigma} d r^{0} \\
& -\int d r^{0} e^{i \omega r^{0}} \int d^{3} \vec{r}^{\prime} \delta^{(3)}\left(\vec{r}^{\prime}-\vec{r}\left(r^{0}\right)\right)\left\{\frac{d r^{0}}{d \sigma} \frac{m}{\pi}\left[\omega^{2} v_{i} v_{j}-i \omega\left(v_{i} \nabla_{j}+v_{j} \nabla_{i}\right)-\frac{\left(1+\vec{v}^{2}\right)}{2}\left(\nabla_{i} \nabla_{j}-\frac{1}{2} \delta_{i j} \nabla^{2}\right)\right]\right. \\
+ & \left.\frac{q Q}{2 \pi M}\left[i \omega\left(v_{i} \nabla_{j}+v_{j} \nabla_{j}-\delta_{i j} v_{k} \nabla_{k}\right)+2\left(\nabla_{i} \nabla_{j}-\frac{1}{2} \delta_{i j} \nabla^{2}\right)\right]\right\} G\left(\omega, \vec{x}, \vec{r}^{\prime}\right), \\
\tilde{a}_{i}(\omega, \vec{x})= & \frac{q}{1+\phi(\vec{x})} \int \frac{e^{i \omega\left(r^{0}+R_{0}\right)}}{4 \pi R_{0}} \frac{v_{i}}{1+\phi(\vec{r})} d r^{0} \\
& -\int d r^{0} e^{i \omega r^{0}} \int d^{3} \vec{r}^{\prime} \delta^{(3)}\left(\vec{r}^{\prime}-\vec{r}\left(r^{0}\right)\right)\left\{\frac{q}{\pi}\left[\omega^{2} v_{i}-i \omega \nabla_{i}+\frac{1}{4} v_{i} \nabla^{2}-\frac{1}{2} v_{k} \nabla_{k} \nabla_{i}\right]\right. \\
& \left.+\frac{d r^{0}}{d \sigma} \frac{4 Q m}{M}\left[v_{k} \nabla_{k} \nabla_{i}+i \omega\left(v_{i} v_{k} \nabla_{k}+\frac{1}{6} \nabla_{i}-\frac{1}{2} \vec{v}^{2} \nabla_{i}\right)\right]\right\} G\left(\omega, \vec{x}, \vec{r}^{\prime}\right),
\end{aligned}
$$

where $\nabla_{i}$ denotes $\frac{\partial}{\partial x^{i}}+\frac{\partial}{\partial r^{i}}$ in Eqs. (2.72) and (2.73). In considering $Q=0$, Eq. (2.72) agrees with [4] and Eq. (2.73) agrees with [2], after taking into account the change in notation and metric signature. The terms involving $Q$ are hence additional contributions due to the RN spacetime. In the following section, we consider the explicit evaluation of the integrals involved in the radiative solutions Eqs. (2.72) and (2.73) in the limit where $\omega \rightarrow 0$. This will enable us to verify that the above results satisfy the soft theorem in the presence of gravitational and electromagnetic interactions and provide the known treelevel soft factors in this case.

\section{SOFT FACTORS AND WAVEFORMS FROM CLASSICAL RADIATION}

In [70], by considering the classical limit of multiple soft theorems in four and higher dimensional spacetimes, the soft factor up to subleading order was related with the power spectrum of low frequency radiation emitted in classical scattering processes. This relation was further used in [71] to unambiguously identify the soft factor in four spacetime dimensions from scattering processes involving a heavy center and a probe particle. The soft factor in this case develops certain logarithmic contributions arising from the long-range gravitational and electromagnetic 
forces experienced by the soft particles. In particular, the result of [4] was shown to satisfy the predicted soft factor and also helped identify an overall phase of the soft radiation. This phase can be understood as arising from the gravitational drag experienced by the soft particle due to the presence of the large scatterer. While the overall phase does not affect the flux of soft particles, it does have implications on the late time gravitational waveform [72]. The soft factor determined from classical scattering processes was shown to be consistent with quantum results [47].

In the following subsections, we will first review the results of $[47,70,71]$. Following these references, we will then evaluate the integrals involved in Eqs. (2.72) and (2.73) in the limit where $\vec{x} \gg \vec{r}(\sigma)$ and when $\omega \rightarrow 0$. The resulting expressions will be shown to provide the correct soft factor involved in the soft photon and soft graviton theorem in the presence of gravitational and electromagnetic interactions. Following the analysis of [72], we investigate the implications of the soft factor results on the memory and tail effect of late-time gravitational and electromagnetic waveforms in the last subsection.

\section{A. Soft factors and evaluation of integrals in four-dimensional spacetimes}

The general relation between the tree-level or classical soft factor and the classical electromagnetic and gravitational radiation in $D$ spacetime dimensions was provided in [70]. Given the trace reversed metric perturbation $\tilde{e}_{\alpha \beta}(\omega, \vec{x})$ and electromagnetic field $\tilde{a}_{a}(\omega, \vec{x})$ in frequency space, we have

$$
\begin{array}{r}
\epsilon^{\alpha \beta} \tilde{e}_{\alpha \beta}(\omega, \vec{x})=\mathcal{N}^{\prime} S_{\mathrm{gr}}(\epsilon, k), \\
\epsilon^{\alpha} \tilde{a}_{\alpha}(\omega, \vec{x})=\mathcal{N}^{\prime} S_{\mathrm{em}}(\epsilon, k), \\
\mathcal{N}^{\prime}=\frac{1}{2 \omega} e^{i \omega R}\left(\frac{\omega}{2 \pi i R}\right)^{\frac{D-2}{2}}, \quad R=|\vec{x}|, \\
\hat{n}=\frac{\vec{x}}{R}, \quad k=-\omega(1, \hat{n}) .
\end{array}
$$

In Eq. (3.1) $\epsilon^{\alpha \beta}$ denotes an arbitrary rank two polarization tensor and $k$ denotes the momentum of the soft graviton, while in Eq. (3.2) $\epsilon^{\alpha}$ denotes an arbitrary polarization vector and $k$ denotes the momentum of the soft photon. $S_{\mathrm{em}}$ and $S_{\mathrm{gr}}$ are the soft factors in the classical limit of the soft graviton theorem and soft photon theorem with the expressions

$$
\begin{aligned}
& S_{\mathrm{gr}}=S_{\mathrm{gr}}^{(0)}+S_{\mathrm{gr}}^{(1)}, \quad S_{\mathrm{em}}=S_{\mathrm{em}}^{(0)}+S_{\mathrm{em}}^{(1)}, \\
& S_{\mathrm{gr}}^{(0)}=\sum_{a=1}^{n} \frac{\epsilon_{\mu \nu} p_{(a)}^{\mu} p_{(a)}^{\nu}}{p_{(a)} \cdot k}, \quad S_{\mathrm{gr}}^{(1)}=i \sum_{a=1}^{n} \frac{\epsilon_{\mu \nu} p_{(a)}^{\mu} k_{\rho}}{p_{(a)} \cdot k} \mathbf{J}_{(a)}^{\rho \nu},
\end{aligned}
$$

$$
\begin{aligned}
S_{\mathrm{em}}^{(0)} & =\sum_{a=1}^{n} q_{(a)} \frac{\epsilon \cdot p_{(a)}}{p_{(a)} \cdot k} \\
S_{\mathrm{em}}^{(1)} & =i \sum_{a=1}^{n} q_{(a)} \frac{\epsilon_{\nu} k_{\rho}}{p_{(a)} \cdot k} \mathbf{J}_{(a)}^{\rho \nu}+\text { nonuniversal. }
\end{aligned}
$$

$S^{(0)}$ and $S^{(1)}$ denote the leading and subleading contributions, respectively, in the soft factors. In the case of the subleading soft photon factor, there do exist nonuniversal contributions and the expression in Eq. (3.5) denotes only the universal piece. The sum in Eqs. (3.4) and (3.5) run over all incoming and outgoing particles, where $q_{(a)} p_{(a)}$ and $\mathbf{J}_{(a)}$ denote the charges, momenta, and angular momenta, counted with a positive sign for incoming particles and a negative sign for outgoing particles.

In [71], the expressions for $S_{\mathrm{gr}}$ and $S_{\mathrm{em}}$ were determined for a system comprising of a probe particle of mass $m$ and charge $q$ scattering off a heavy central object of either mass $M$ or charge $Q$ in four spacetime dimensions. The result for $S_{\mathrm{gr}}$ in the case of only gravitational interactions was also shown to agree with [4] after evaluating the integrals involved in the classical solutions. We will now review this procedure and apply it subsequently to the gravitational and electromagnetic radiative solutions on the RN spacetime.

The expression for $S_{\mathrm{gr}}$ in Eq. (3.1) is invariant under $\epsilon^{\mu \nu} \rightarrow \epsilon^{\mu \nu}+\xi^{\mu} k^{\nu}+\xi^{\nu} k^{\mu}$, where $\xi^{\alpha}$ is arbitrary. This implies the constraint $k^{\mu} \tilde{e}_{\mu \nu}=0$ for the radiative components of the gravitational field. We can hence determine the $\tilde{e}_{0 \nu}$ components from $\tilde{e}_{i j}$ and set $\epsilon^{0 \nu}=0$. Similarly, $S_{\mathrm{em}}$ in Eq. (3.2) is invariant under $\epsilon^{\mu} \rightarrow \epsilon^{\mu}+k^{\mu}$. The constraint $k^{\mu} \tilde{a}_{\mu}=0$ on the radiative components imply that $\tilde{a}_{0}$ can be determined from $\tilde{a}_{i}$ and that we can set $\epsilon^{0}=0$. We thus have

$$
\epsilon^{0 \nu}=0, \quad \epsilon^{0}=0 .
$$

In addition, the expressions for $\tilde{e}_{\mu \nu}$ and $\tilde{a}_{\mu}$ are known only up to a choice in gauge. Denoting the arbitrary gauge parameters by $\xi_{\mu}$ and $\xi$ (for gravity) and $\lambda$ (for electromagnetism), we have the following gauge transformations:

$$
\delta \tilde{e}_{\mu \nu}=k_{\mu} \xi_{\nu}+k_{\nu} \xi_{\mu}-\xi . k \eta_{\mu \nu}, \quad \delta \tilde{a}_{\mu}=\lambda k_{\mu}
$$

Using Eq. (3.7) in Eqs. (3.1) and (3.2), we then have the relations

$$
k_{\mu} \epsilon^{\mu \nu}-\frac{1}{2} k^{\nu} \epsilon_{\mu}^{\mu}=0, \quad k_{\mu} \epsilon^{\mu}=0
$$

Equations (3.8) and (3.6) then provide the following conditions on the polarization tensor in Eq. (3.1) and the polarization vector in Eq. (3.2) 
$\epsilon^{0 \nu}=0, \quad k_{i} \epsilon^{i j}=0, \quad \epsilon_{i}^{i}=0 ; \quad \epsilon^{0}=0, \quad k_{i} \epsilon^{i}=0$.

To determine $S_{\mathrm{gr}}$ and $S_{\mathrm{em}}$, we also need to determine the expressions for the momenta and angular momenta. Due to the presence of the long range interaction of electromagnetic and gravitational forces, the particle trajectories of the probe before $\left(r_{(1)}\right)$ and after $\left(r_{(2)}\right)$, the scattering can be taken to have the form

$$
\begin{aligned}
& r_{(1)}^{0}=t=r_{(2)}^{0}, \quad \vec{r}_{(1)}=\vec{\beta}_{-} t+\vec{c}_{-}-C_{-} \vec{\beta}_{-} \ln |t|, \\
& \vec{r}_{(2)}=\vec{\beta}_{+} t+\vec{c}_{+}-C_{+} \vec{\beta}_{+} \ln |t|,
\end{aligned}
$$

where the $\ln |t|$ terms are the contributions to the long range forces. The momenta of the probe particle before and after scattering will be denoted by $p_{(1)}$ and $p_{(2)}$, respectively, while the angular momenta of the probe before and after scattering will be denoted by $\mathbf{j}_{(1)}^{\mu \nu}$ and $\mathbf{j}_{(2)}^{\mu \nu}$, respectively. Using Eq. (3.10) and retaining all $\ln |t|$ terms, the following asymptotic expressions for the momenta and angular momenta can be determined:

$$
\begin{aligned}
& p_{(1)}=\frac{m}{\sqrt{1-\vec{\beta}_{-}^{2}}}\left(1, \vec{\beta}_{-}\right), \quad p_{(2)}=-\frac{m}{\sqrt{1-\vec{\beta}_{+}^{2}}}\left(1, \vec{\beta}_{+}\right), \\
& \mathbf{j}_{(1)}^{i j}=r_{(1)}^{i} p_{(1)}^{j}-r_{(1)}^{j} p_{(1)}^{i}=\frac{m}{\sqrt{1-\vec{\beta}_{-}^{2}}}\left(c_{-}^{i} \beta_{-}^{j}-c_{-}^{j} \beta_{-}^{i}\right), \\
& \mathbf{j}_{(1)}^{0 i}=r_{(1)}^{0} p_{(1)}^{i}-r_{(1)}^{i} p_{(1)}^{0}=-\frac{m}{\sqrt{1-\vec{\beta}_{-}^{2}}}\left(c_{-}^{i}-C_{-} \beta_{-}^{i} \ln |t|\right), \\
& \mathbf{j}_{(2)}^{i j}=r_{(2)}^{i} p_{(2)}^{j}-r_{(2)}^{j} p_{(2)}^{i}=-\frac{m}{\sqrt{1-\vec{\beta}_{+}^{2}}}\left(c_{+}^{i} \beta_{+}^{j}-c_{+}^{j} \beta_{+}^{i}\right), \\
& \mathbf{j}_{(2)}^{0 i}=r_{(2)}^{0} p_{(2)}^{i}-r_{(2)}^{i} p_{(2)}^{0}=\frac{m}{\sqrt{1-\vec{\beta}_{+}^{2}}}\left(c_{+}^{i}-C_{+} \beta_{+}^{i} \ln |t|\right) .
\end{aligned}
$$

Substituting Eqs. (3.9) and (3.11) and the expression for $k$ from Eq. (3.3) in Eqs. (3.1) and (3.2), and replacing $\ln |t|$ with $\ln \omega^{-1}$, gives the expressions for the soft factors in the probe-scatterer approximation up to overall phases

$$
\begin{aligned}
S_{\mathrm{em}}=-\frac{q}{\omega}\left[\frac{\vec{\epsilon} \cdot \vec{\beta}_{+}}{1-\hat{n} \cdot \vec{\beta}_{+}}-\frac{\vec{\epsilon} \cdot \vec{\beta}_{-}}{1-\hat{n} \cdot \vec{\beta}_{-}}\right]-i q \ln \omega^{-1}\left[C_{+} \frac{\vec{\epsilon} \cdot \vec{\beta}_{+}}{1-\hat{n} \cdot \vec{\beta}_{+}}-C_{-} \frac{\vec{\epsilon} \cdot \vec{\beta}_{-}}{1-\hat{n} \cdot \vec{\beta}_{-}}\right]+\text {finite, } \\
S_{\mathrm{gr}}=-\frac{m}{\omega} \epsilon^{i j}\left[\frac{1}{1-\hat{n} \cdot \vec{\beta}_{+}} \frac{1}{\sqrt{1-\vec{\beta}_{+}^{2}}} \beta_{+i} \beta_{+j}-\frac{1}{1-\hat{n} \cdot \vec{\beta}_{-}} \frac{1}{\sqrt{1-\vec{\beta}_{-}^{2}}} \beta_{-i} \beta_{-j}\right] \\
-i m \ln \omega^{-1} \epsilon^{i j}\left[C_{+} \frac{1}{1-\hat{n} \cdot \vec{\beta}_{+}} \frac{1}{\sqrt{1-\vec{\beta}_{+}^{2}}} \beta_{+i} \beta_{+j}-C_{-} \frac{1}{1-\hat{n} \cdot \vec{\beta}_{-}} \frac{1}{\sqrt{1-\vec{\beta}_{-}^{2}}} \beta_{-i} \beta_{-j}\right]+\text { finite. }
\end{aligned}
$$

The substitution of $\ln |t|$ with $\ln \omega^{-1}$ was confirmed by comparison with classical scattering examples. This included the solution for gravitational radiation given in [4], which further provided evidence for an overall phase present in the soft factor arising from the backscattering of soft gravitons due to the potential of the central scatterer. The presence of $\ln \omega^{-1}$ in the quantum subleading soft factors and a first principle account for the overall phase in four dimensions were provided in [47]. The classical result therein involves a covariant generalization beyond the probe-scatterer approximation in the presence of both gravitational and electromagnetic interactions. The phase term for the low frequency gravitational waves was argued to arise as a consequence of logarithmic corrections in the trajectory of the soft particle. Specifically, the soft particle trajectory is

$$
x^{\mu}(\tau)=n^{\mu}(\tau)+m^{\mu} \ln |\tau|
$$

where $\tau$ its affine parameter associated with the trajectory $n=(1, \hat{n})$ is a null vector along the asymptotic direction of motion of the soft particle and $m^{\mu}$ is a four vector, which in the case of a heavy central scatterer of mass $M$ is given by

$$
m^{\mu}=\frac{M}{4 \pi}(1, \overrightarrow{0})
$$

By using the equation of motion of the soft particle, the analysis of [47] identifies the overall phase

$$
\exp [i \theta]=\exp [i k \cdot m \ln (R \omega)]
$$

where $R$ is the distance of the soft particle from the scattering center. This is the common overall phase which multiplies the soft photon and graviton factors, which in the probe-scatterer approximation becomes $\exp \left[i \frac{M}{4 \pi} \omega \ln (R \omega)\right]$. By expanding the exponent and keeping terms of order $\omega \ln (R \omega)$, one finds a phase correction at the subleading 
soft photon and graviton factors. The corrections to Eq. (3.12) and Eq. (3.13) are

$$
\begin{gathered}
\Delta S_{\mathrm{em}}=-i q \frac{M}{4 \pi} \ln (R \omega)\left[\frac{\vec{\epsilon} \cdot \vec{\beta}_{+}}{1-\hat{n} \cdot \vec{\beta}_{+}}-\frac{\vec{\epsilon} \cdot \vec{\beta}_{-}}{1-\hat{n} \cdot \vec{\beta}_{-}}\right] \\
\Delta S_{\mathrm{gr}}=-i m \frac{M}{4 \pi} \ln (R \omega) \epsilon^{i j}\left[\frac{1}{1-\hat{n} \cdot \vec{\beta}_{+}} \frac{1}{\sqrt{1-\vec{\beta}_{+}^{2}}} \beta_{+i} \beta_{+j}\right. \\
\left.-\frac{1}{1-\hat{n} \cdot \vec{\beta}_{-}} \frac{1}{\sqrt{1-\vec{\beta}_{-}^{2}}} \beta_{-i} \beta_{-j}\right] .
\end{gathered}
$$

The expressions for $S_{\mathrm{em}}+\Delta S_{\mathrm{em}}$ and $S_{\mathrm{gr}}+\Delta S_{\mathrm{gr}}$, using Eqs. (3.12), (3.13), (3.18), and (3.17), agree with the soft factor expressions of [47] in the probe-scatterer approximation.

In considering the classical expressions for the radiative fields, we will need to evaluate certain integrals of the type

$$
I=\int d t e^{i \omega g(t)} F(t)+\text { boundary terms, }
$$

where $g(t) \rightarrow a_{ \pm} t+b_{ \pm} \ln |t|$ and $F(t)$ is either a constant or falls off as a negative power of $t$ as $t \rightarrow \infty$. If $F(t) \sim|t|^{-\alpha}$ with $-1<\alpha \leq 0[\alpha=0$ is the case where $F(t)$ is a constant] then the integral in Eq. (3.19) has to be defined by first performing an integration by parts using the identity $e^{i \omega g(t)}=\frac{1}{i \omega g^{\prime}(t)} \frac{d}{d t} e^{i \omega g(t)}$, where $g^{\prime}(t)=\frac{d}{d t} g(t)$. This gives

$$
I=\int d t \frac{1}{i \omega g^{\prime}(t)} \frac{d}{d t} e^{i \omega g(t)} F(t)+\text { boundary terms }=-\frac{1}{i \omega} \int d t e^{i \omega g(t)} \frac{d}{d t}\left(\frac{F(t)}{i \omega g^{\prime}(t)}\right) .
$$

In evaluating the integrals, we will make specific use of the following five integral relations derived in [71],

$$
\begin{aligned}
& I_{1}=\frac{1}{\omega} \int_{-\infty}^{+\infty} d t e^{-i \omega g(t)} \frac{d}{d t} f(t)=\frac{1}{\omega}\left(f_{+}-f_{-}\right)+i\left(a_{+} k_{+}-a_{-} k_{-}\right) \ln \omega^{-1}+\text { finite, } \\
& I_{2}=\int_{-\infty}^{+\infty} d t e^{-i \omega g(t)} \frac{d}{d t}\left[f(t)\left(\ln \frac{h(t)}{R}+\int_{h(t)}^{+\infty} d u \frac{e^{i \omega u}}{u}\right)\right]=-\left(f_{+}-f_{-}\right) \ln (R \omega)+\text { finite, } \\
& I_{3}=\int_{-\infty}^{+\infty} d t \frac{f(t)}{r(t)}\left(e^{-i \omega g(t)}-e^{-i \omega h(t)}\right)=\text { finite, } \\
& I_{4}=\frac{1}{\omega} \int_{-\infty}^{+\infty} d t \frac{f(t)}{r(t)^{2}}\left(e^{-i \omega g(t)}-e^{-i \omega h(t)}\right)=-i\left(\frac{f_{+}}{c_{+}^{2}}\left(a_{+}-p_{+}\right)-\frac{f_{-}}{c_{-}^{2}}\left(a_{-}-p_{-}\right)\right) \ln \omega^{-1}+\text { finite, } \\
& I_{5}=\int_{-\infty}^{+\infty} d t \frac{f(t)}{r(t)} e^{-i \omega g(t)}=\left(\frac{f_{+}}{c_{+}}-\frac{f_{-}}{c_{-}}\right) \ln \omega^{-1}+\text { finite, }
\end{aligned}
$$

where

$$
\begin{aligned}
& f(t) \rightarrow f_{ \pm}+\frac{k_{ \pm}}{t}, \quad g(t) \rightarrow a_{ \pm} t+b_{ \pm} \ln |t|, \\
& h(t) \rightarrow p_{ \pm} t+q_{ \pm} \ln |t|, \quad r(t) \rightarrow c_{ \pm} t+d_{ \pm} \ln |t|, \quad \text { as } t \rightarrow \infty .
\end{aligned}
$$

In Eq. (3.21) " $R$ " is an arbitrary constant and "finite" represents terms which are finite in the $\omega \rightarrow 0$ limit. The integral relations in Eq. (3.21) were used to demonstrate that the result of [4] agrees with the graviton soft factor. In the following subsections, we will demonstrate that the photon and graviton soft factors are also satisfied by the probe particle scattering results on the RN spacetime.

\section{B. Electromagnetic soft factors and phases on the RN spacetime}

In this subsection we will evaluate Eq. (2.73) in order to determine the soft factor expression. Using Eq. (3.2), the normalization in $D=4$ dimensions from Eq. (3.3) and the polarization conditions Eq. (3.9), we have the following four-dimensional expression for the electromagnetic soft factor:

$$
S_{\mathrm{em}}=i \frac{4 \pi R}{e^{i \omega R}} \epsilon^{i} \tilde{a}_{i}(\omega, \vec{x}) .
$$

The integrals in Eq. (2.73) will be evaluated in the limit where the electromagnetic waves are far separated from the probe particle, i.e., $\vec{x} \gg \vec{r}(\sigma)$. In this limit, the expression of $G_{M}(\omega, \vec{x}, \vec{r})$ in Eq. (2.59) simplifies to 


$$
\lim _{\vec{x} \gg \vec{r}} G_{M}(\omega, \vec{x}, \vec{r}) \rightarrow \frac{i M}{16 \pi \omega}\left[\ln \left(\frac{|\vec{r}|+\hat{n} . \vec{r}}{R}\right)+\int_{|\vec{r}|+\hat{n} . \vec{r}}^{\infty} \frac{d u}{u} e^{i \omega u}\right] \frac{e^{i \omega(R-\hat{n} . \vec{r})}}{R}:=\tilde{G}_{M}(\omega, \vec{x}, \vec{r}) .
$$

Setting $r^{0}=t$ and using Eq. (3.24) in Eq. (2.73), we find that the solutions for electromagnetic perturbations in Eq. (2.73) take the form

$$
\begin{aligned}
\tilde{a}_{i}(\omega, \vec{x})= & \tilde{a}_{i}^{(1)}(\omega, \vec{x})+\tilde{a}_{i}^{(2)}(\omega, \vec{x})+\tilde{a}_{i}^{(3)}(\omega, \vec{x})+\tilde{a}_{i}^{(4)}(\omega, \vec{x})+\tilde{a}_{i}^{(5)}(\omega, \vec{x})+\tilde{a}_{i}^{(6)}(\omega, \vec{x}) \\
& +\tilde{a}_{i}^{(7)}(\omega, \vec{x})+\tilde{a}_{i}^{(8)}(\omega, \vec{x})+\tilde{a}_{i}^{(9)}(\omega, \vec{x})
\end{aligned}
$$

where

$$
\begin{aligned}
& \tilde{a}_{i}^{(1)}(\omega, \vec{x})=\frac{q}{4 \pi} \frac{e^{i \omega R}}{R} \int d t \frac{e^{i \omega(t-\hat{n} . \vec{r})}}{1+\phi(\vec{r}(t))} v_{i} \\
& \tilde{a}_{i}^{(2)}(\omega, \vec{x})=\frac{q}{2 \pi} \int d t e^{i \omega t} v_{k} \nabla_{k} \nabla_{i} \tilde{G}_{M}(\omega, \vec{x}, \vec{r}), \\
& \tilde{a}_{i}^{(3)}(\omega, \vec{x})=\frac{i \omega q}{\pi} \int d t e^{i \omega t} \nabla_{i} \tilde{G}_{M}(\omega, \vec{x}, \vec{r}), \\
& \tilde{a}_{i}^{(4)}(\omega, \vec{x})=-\frac{i M q \omega}{16 \pi^{2}} \frac{e^{i \omega R}}{R} \int d t v_{i}\left\{\ln \frac{\left|\vec{r}^{\prime}\right|+\hat{n} \cdot \vec{r}^{\prime}}{R} e^{i \omega\left(t-\hat{n} \cdot \vec{r}^{\prime}\right)}+\int_{\left|\vec{r}^{\prime}\right|+\hat{n} . \vec{r}^{\prime}}^{\infty} \frac{d u}{u} e^{i \omega\left(t-\hat{n} . \vec{r}^{\prime}+u\right)}\right\}, \\
& \tilde{a}_{i}^{(5)}(\omega, \vec{x})=-\frac{q}{4 \pi} \int d t e^{i \omega t} v_{i} \nabla_{k} \nabla_{k} \tilde{G}_{M}(\omega, \vec{x}, \vec{r}), \\
& \tilde{a}_{i}^{(6)}(\omega, \vec{x})=-\frac{4 Q m}{M} \int d t \frac{d t}{d \sigma} e^{i \omega t} v_{k} \nabla_{k} \nabla_{i} \tilde{G}_{M}(\omega, \vec{x}, \vec{r}), \\
& \tilde{a}_{i}^{(7)}(\omega, \vec{x})=-\frac{i \omega 4 Q m}{M} \int d t \frac{d t}{d \sigma} e^{i \omega t} v_{i} v_{k} \nabla_{k} \tilde{G}_{M}(\omega, \vec{x}, \vec{r}) \\
& \tilde{a}_{i}^{(8)}(\omega, \vec{x})=-\frac{i \omega 2 Q m}{3 M} \int d t \frac{d t}{d \sigma} e^{i \omega t} \nabla_{i} \tilde{G}_{M}(\omega, \vec{x}, \vec{r}), \\
& \tilde{a}_{i}^{(9)}(\omega, \vec{x})=\frac{i \omega 2 Q m}{M} \int d t \frac{d t}{d \sigma} e^{i \omega t} \vec{v}^{2} \nabla_{i} \tilde{G}_{M}(\omega, \vec{x}, \vec{r}),
\end{aligned}
$$

where $\nabla_{i}=\frac{\partial}{\partial r^{i}}+\frac{\partial}{\partial x^{i}}$. Apart from the terms $\tilde{a}_{i}^{(6)}(\omega, \vec{x}), \cdots \tilde{a}_{i}^{(9)}(\omega, \vec{x})$ which involve $Q$, the terms $\tilde{a}_{i}^{(1)}(\omega, \vec{x}), \cdots \tilde{a}_{i}^{(5)}(\omega, \vec{x})$ are the same as those which arise for a probe charge on the Schwarzschild spacetime [2], after accounting for the difference in notation and metric signature.

The integrals can be evaluated using the expressions in Eq. (3.21) and the asymptotic expressions for the particle trajectory and its velocity given in Eq. (3.10). This analysis has been provided in detail in Appendix C. We find that only the terms $\tilde{a}_{i}^{(1)}(\omega, \vec{x}), \tilde{a}_{i}^{(4)}(\omega, \vec{x})$, and $\tilde{a}_{i}^{(5)}(\omega, \vec{x})$ involve coefficients of $\omega^{-1}$ and $\ln \omega^{-1}$. The results for the integrals in Eqs. (3.26), (3.29), and (3.30) in the soft limit are

$$
\begin{aligned}
\tilde{a}_{i}^{(1)}(\omega, \vec{x})= & i \frac{q}{\omega} \frac{e^{i \omega R}}{4 \pi R}\left(\frac{1}{1-\hat{n} \cdot \vec{\beta}_{+}} \beta_{+i}-\frac{1}{1-\hat{n} \cdot \vec{\beta}_{-}} \beta_{-}^{i}\right) \\
& -q \ln \omega^{-1} \frac{e^{i \omega R}}{4 \pi R}\left(\frac{C_{+}}{1-\hat{n} \cdot \vec{\beta}_{+}} \beta_{+i}-\frac{C_{-}}{1-\hat{n} \cdot \vec{\beta}_{-}} \beta_{-i}-\frac{M}{8 \pi\left|\vec{\beta}_{+}\right|} \beta_{+i}-\frac{M}{8 \pi\left|\vec{\beta}_{-}\right|} \beta_{-i}\right),
\end{aligned}
$$




$$
\begin{gathered}
\tilde{a}_{i}^{(4)}(\omega, \vec{x})=-M q \frac{e^{i \omega R}}{16 \pi^{2} R} \ln (\omega R)\left(\frac{1}{1-\hat{n} \cdot \vec{\beta}_{+}} \beta_{+i}-\frac{1}{1-\hat{n} \cdot \vec{\beta}_{-}} \beta_{-i}\right)+\text { finite } \\
\tilde{a}_{i}^{(5)}(\omega, \vec{x})=-M q \frac{e^{i \omega R}}{32 \pi^{2} R}\left(\frac{\beta_{+i}}{\left|\vec{\beta}_{+}\right|}-\frac{\beta_{-i}}{\left|\vec{\beta}_{-}\right|}\right) \ln \omega^{-1}+\text { finite. }
\end{gathered}
$$

In particular, the result for $\tilde{a}_{i}^{(4)}$ in Eq. (3.36) will provide the phase contribution to the soft factor expression. We can now substitute Eqs. (3.35)-(3.37) in Eq. (3.23) to find the following soft factor expression for the electromagnetic radiation:

$$
\begin{aligned}
S_{\mathrm{em}}= & i \frac{4 \pi R}{e^{i \omega R}} \epsilon^{i} \tilde{a}_{i} \\
= & -\frac{q}{\omega}\left(\frac{\vec{\epsilon} \cdot \vec{\beta}_{+}}{1-\hat{n} \cdot \vec{\beta}_{+}}-\frac{\vec{\epsilon} \cdot \vec{\beta}_{-}}{1-\hat{n} \cdot \vec{\beta}_{-}}\right)-i q \ln \omega^{-1}\left(\frac{C_{+}}{1-\hat{n} \cdot \vec{\beta}_{+}} \vec{\epsilon} \cdot \vec{\beta}_{+}-\frac{C_{-}}{1-\hat{n} \cdot \overrightarrow{\beta_{-}}} \vec{\epsilon} \cdot \vec{\beta}_{-}\right) \\
& -i q \frac{M}{4 \pi} \ln (\omega R)\left(\frac{\vec{\epsilon} \cdot \vec{\beta}_{+}}{1-\hat{n} \cdot \vec{\beta}_{+}}-\frac{\vec{\epsilon} \cdot \vec{\beta}_{-}}{1-\hat{n} \cdot \vec{\beta}_{-}}\right) .
\end{aligned}
$$

The second line of Eq. (3.38) agrees with the expression for the soft factor in Eq. (3.12), while the third line in Eq. (3.38) agrees with the predicted phase contribution to the soft factor in Eq. (3.17) resulting from the backscattering of soft photons due to the gravitational potential of the RN black hole.

\section{Gravitational soft factors and phases on the RN spacetime}

We will now carry out the analysis of the previous subsection on the solutions for gravitational perturbations in Eq. (2.72). Using Eqs. (3.1), (3.3), and (3.9), we find the following expression for $S_{\mathrm{gr}}$ in $D=4$ dimensions:

$$
S_{\mathrm{gr}}=i \frac{4 \pi R}{e^{i \omega R}} \epsilon^{i j} \tilde{e}_{i j}(\omega, \vec{x}) .
$$

As in the electromagnetic case, we let $r^{0}=t$ and $x \gg r(t)$ by substituting Eq. (3.24) in Eq. (2.72). The resulting expression for Eq. (2.72) can be expressed as

$$
\tilde{e}_{i j}(\omega, \vec{x})=\tilde{e}_{i j}^{(1)}(\omega, \vec{x})+\tilde{e}_{i j}^{(2)}(\omega, \vec{x})+\tilde{e}_{i j}^{(3)}(\omega, \vec{x})+\tilde{e}_{i j}^{(4)}(\omega, \vec{x})+\tilde{e}_{i j}^{(5)}(\omega, \vec{x})+\tilde{e}_{i j}^{(6)}(\omega, \vec{x})+\tilde{e}_{i j}^{(7)}(\omega, \vec{x}),
$$

where

$$
\begin{gathered}
\tilde{e}_{i j}^{(1)}(\omega, \vec{x})=\frac{m e^{i \omega R}}{4 \pi R} \int \frac{d t}{(1+2 \Phi(\vec{r}(t)))} \frac{d t}{d \sigma} v_{i} v_{j} e^{i \omega(t-\hat{n} \cdot \vec{r}(t))}+\text { boundary terms, } \\
\tilde{e}_{i j}^{(2)}(\omega, \vec{x})=\frac{m}{2 \pi} \int d t \frac{d t}{d \sigma} e^{i \omega t}\left(1+\vec{v}^{2}\right)\left(\nabla_{i} \nabla_{j}-\frac{1}{2} \delta_{i j} \nabla_{k} \nabla_{k}\right) \tilde{G}_{M}(\omega, \vec{x}, \vec{r}), \\
\tilde{e}_{i j}^{(3)}(\omega, \vec{x})=-i \frac{M m}{16 \pi^{2}} \omega \frac{e^{i \omega R}}{R} \int d t \frac{d t}{d \sigma} v_{i} v_{j}\left\{\ln \frac{\left|\vec{r}^{\prime}\right|+\hat{n} \cdot \vec{r}^{\prime}}{R} e^{i \omega\left(t-\hat{n} \cdot \vec{r}^{\prime}\right)}+\int_{\left|\vec{r}^{\prime}\right|+\hat{n} \cdot \vec{r}^{\prime}}^{\infty} \frac{d u}{u} e^{i \omega\left(t-\hat{n} \cdot \vec{r}^{\prime}+u\right)}\right\}, \\
\tilde{e}_{i j}^{(4)}(\omega, \vec{x})=\frac{i \omega m}{\pi} \int d t \frac{d t}{d \sigma} e^{i \omega t}\left(v_{i} \nabla_{j}+v_{j} \nabla_{i}\right) \tilde{G}_{M}(\omega, \vec{x}, \vec{r}), \\
\tilde{e}_{i j}^{(5)}(\omega, \vec{x})=-i \omega \frac{q Q}{2 \pi M} \int d t e^{i \omega t} \delta_{i j} v^{k} \nabla_{k} \tilde{G}_{M}(\omega, \vec{x}, \vec{r}), \\
\tilde{e}_{i j}^{(6)}(\omega, \vec{x})=i \frac{q Q}{\pi M} \int d t e^{i \omega t}\left(\nabla_{i} \nabla_{j}-\frac{1}{2} \delta_{i j} \nabla_{k} \nabla_{k}\right) \tilde{G}_{M}(\omega, \vec{x}, \vec{r}),
\end{gathered}
$$




$$
\tilde{e}_{i j}^{(7)}(\omega, \vec{x})=i \omega \frac{q Q}{2 \pi M} \int d t e^{i \omega t}\left(v_{i} \nabla_{j}+v_{j} \nabla_{i}\right) \tilde{G}_{M}(\omega, \vec{x}, \vec{r})
$$

The $\tilde{e}_{i j}^{(1)}(\omega, \vec{x}) \cdots \tilde{e}_{i j}^{(4)}(\omega, \vec{x})$ contributions are those of the Schwarzschild spacetime. The evaluation of these integrals in the soft limit has been provided in Appendix C. Only four of the above integrals, $\tilde{e}_{i j}^{(1)}(\omega, \vec{x}), \tilde{e}_{i j}^{(2)}(\omega, \vec{x}), \tilde{e}_{i j}^{(3)}(\omega, \vec{x})$, and $\tilde{e}_{i j}^{(6)}(\omega, \vec{x})$, contribute in the soft limit with expressions

$$
\begin{aligned}
& \tilde{e}_{i j}^{(1)}(\omega, \vec{x})=i \frac{m}{4 \pi \omega} \frac{e^{i \omega R}}{R}\left\{\frac{1}{1-\hat{n} \cdot \vec{\beta}_{+}} \frac{1}{\sqrt{1-\vec{\beta}_{+}^{2}}} \beta_{+i} \beta_{+j}-\frac{1}{1-\hat{n} \cdot \vec{\beta}_{-}} \frac{1}{\sqrt{1-\vec{\beta}_{-}^{2}}} \beta_{-i} \beta_{-j}\right\} \\
& -\frac{m}{4 \pi R} e^{i \omega R} \ln \omega^{-1}\left[\frac{1}{\sqrt{1-\vec{\beta}_{+}^{2}}} \beta_{+i} \beta_{+j}\left\{C_{+} \frac{1}{1-\hat{n} \cdot \vec{\beta}_{+}}-\frac{M_{0}}{8 \pi\left|\vec{\beta}_{+}\right|} \frac{3-\vec{\beta}_{+}^{2}}{1-\vec{\beta}_{+}^{2}}+C_{+} \frac{1}{1-\vec{\beta}_{+}^{2}}\right\}\right. \\
& \left.-\frac{1}{\sqrt{1-\vec{\beta}_{-}^{2}}} \beta_{-i} \beta_{-j}\left\{C_{-} \frac{1}{1-\hat{n} \cdot \vec{\beta}_{-}}+\frac{M_{0}}{8 \pi\left|\vec{\beta}_{-}\right|} \frac{3-\vec{\beta}_{-}^{2}}{1-\vec{\beta}_{-}^{2}}+C_{-} \frac{1}{1-\vec{\beta}_{-}^{2}}\right\}\right] \text {, } \\
& \tilde{e}_{i j}^{(2)}(\omega, \vec{x})=-M m \frac{e^{i \omega R}}{32 \pi^{2} R} \ln \omega^{-1}\left[\frac{\left(1+\vec{\beta}_{+}^{2}\right) \beta_{+i} \beta_{+j}}{\left|\vec{\beta}_{+}\right|^{3} \sqrt{1-\vec{\beta}_{+}^{2}}}+\frac{\left(1+\vec{\beta}_{-}^{2}\right) \beta_{-i} \beta_{-j}}{\left|\vec{\beta}_{-}\right|^{3} \sqrt{1-\vec{\beta}_{-}^{2}}}\right]+\text { finite } \\
& \tilde{e}_{i j}^{(3)}(\omega, \vec{x})=-\frac{M m}{16 \pi^{2}} \ln (\omega R) \frac{e^{i \omega R}}{R}\left\{\frac{1}{1-\hat{n} \cdot \vec{\beta}_{+}} \frac{1}{\sqrt{1-\vec{\beta}_{+}^{2}}} \beta_{+i} \beta_{+j}-\frac{1}{1-\hat{n} \cdot \vec{\beta}_{-}} \frac{1}{\sqrt{1-\vec{\beta}_{-}^{2}}} \beta_{-i} \beta_{-j}\right\}, \\
& \tilde{e}_{i j}^{(6)}(\omega, \vec{x})=-Q q \frac{e^{i \omega R}}{16 \pi^{2} R} \ln \omega^{-1}\left[\frac{\beta_{+i} \beta_{+j}}{\left|\vec{\beta}_{+}\right|^{3}}+\frac{\beta_{-i} \beta_{-j}}{\left|\vec{\beta}_{-}\right|^{3}}\right]+\text { finite. }
\end{aligned}
$$

All other integrals do not contribute any terms involving $\omega^{-1}$ and $\ln \omega^{-1}$ terms. Substituting Eqs. (3.48)-(3.51) in Eq. (3.39) gives

$$
\begin{aligned}
S_{\mathrm{gr}}= & i \frac{4 \pi R}{e^{i \omega R}} \epsilon^{i j} \tilde{e}_{i j}(\omega, \vec{x}) \\
= & -\frac{m}{\omega} \epsilon^{i j}\left[\frac{1}{1-\hat{n} \cdot \vec{\beta}_{+}} \frac{1}{\sqrt{1-\vec{\beta}_{+}^{2}}} \beta_{+i} \beta_{+j}-\frac{1}{1-\hat{n} \cdot \vec{\beta}_{-}} \frac{1}{\sqrt{1-\vec{\beta}_{-}^{2}}} \beta_{-i} \beta_{-j}\right] \\
& -i m \ln \omega^{-1} \epsilon^{i j}\left[\frac{1}{\sqrt{1-\vec{\beta}_{+}^{2}}}\left\{C_{+}\left(\frac{1}{1-\hat{n} \cdot \vec{\beta}_{+}}+\frac{1}{1-\vec{\beta}_{+}^{2}}\right)-\frac{M}{8 \pi\left|\vec{\beta}_{+}\right|^{3}} \frac{3 \vec{\beta}_{+}^{2}-1}{1-\vec{\beta}_{+}^{2}}\right\} \beta_{+i} \beta_{+j}\right. \\
& \left.-\frac{1}{\sqrt{1-\vec{\beta}_{-}^{2}}}\left\{C_{-}\left(\frac{1}{1-\hat{n} \cdot \vec{\beta}_{-}}+\frac{1}{1-\vec{\beta}_{-}^{2}}\right)+\frac{M}{8 \pi\left|\vec{\beta}_{-}\right|^{3}} \frac{3 \vec{\beta}_{-}^{2}-1}{1-\vec{\beta}_{-}^{2}}\right\} \beta_{-i} \beta_{-j}\right] \\
& -i m \frac{M}{4 \pi} \ln (R \omega) \epsilon^{i j}\left[\frac{1}{1-\hat{n}_{3} \cdot \vec{\beta}_{+}} \frac{1}{\sqrt{1-\vec{\beta}_{+}^{2}}} \beta_{+i} \beta_{+j}-\frac{1}{1-\hat{n} \cdot \vec{\beta}_{-}} \frac{1}{\sqrt{1-\vec{\beta}_{-}^{2}}} \beta_{-i} \beta_{-j}\right] \\
- & i \frac{q Q}{4 \pi} \ln \omega^{-1} \epsilon^{i j}\left[\frac{\beta_{+i} \beta_{+j}}{\left|\vec{\beta}_{+}\right|^{3}}+\frac{\beta_{-i} \beta_{-j}}{\left|\vec{\beta}_{-}\right|^{3}}\right]+\text { finite. }
\end{aligned}
$$

The leading contribution in the above expression agrees with Eq. (3.13) and the phase correction agrees with Eq. (3.18). To complete the agreement with the subleading term in Eq. (3.13), we need the expression for $C_{ \pm}$. This can be determined by considering the energy of the probe particle from the point particle action in Eq. (2.5), 


$$
-E=\frac{\delta S_{p p}}{\delta\left(\frac{d t}{d \sigma}\right)}=-m\left|g_{00}\right| \frac{d t}{d \sigma}+\frac{q}{4 \pi} A_{0}
$$

The asymptotic form of Eq. (3.53) can be determined using Eqs. (2.22), (C1), (C3), and Eq. (C4). We find

$$
-m\left|g_{00}\right| \frac{d t}{d \sigma}+\frac{q}{4 \pi} A_{0}=-\frac{m}{\sqrt{1-\vec{\beta}_{ \pm}^{2}}}\left[1-\frac{1}{t}\left(\frac{C_{ \pm} \vec{\beta}_{ \pm}^{2}}{1-\vec{\beta}_{ \pm}^{2}} \mp \frac{M}{8 \pi\left|\vec{\beta}_{ \pm}\right|}\left(\frac{3 \vec{\beta}_{ \pm}^{2}-1}{1-\vec{\beta}_{ \pm}^{2}}\right)\right)\right] \pm \frac{1}{t} \frac{q Q}{\left|\vec{\beta}_{ \pm}\right|}+\mathcal{O}\left(t^{-2}\right) .
$$

Requiring a constant asymptotic energy, we can set the $\frac{1}{t}$ coefficient in Eq. (3.54) to vanish. This provides the following expression relating $C_{ \pm}$with $M$ and $Q$ :

$$
C_{ \pm}= \pm \frac{M}{8 \pi\left|\vec{\beta}_{ \pm}\right|^{3}}\left(3 \vec{\beta}_{ \pm}^{2}-1\right) \mp \frac{q Q}{4 \pi m\left|\vec{\beta}_{ \pm}\right|^{3}}\left(1-\vec{\beta}_{ \pm}^{2}\right)^{3 / 2}
$$

Substituting this expression in (3.52) then gives

$$
\begin{aligned}
S_{\mathrm{gr}}= & -\frac{m}{\omega} \epsilon^{i j}\left[\frac{1}{1-\hat{n} \cdot \vec{\beta}_{+}} \frac{1}{\sqrt{1-\vec{\beta}_{+}^{2}}} \beta_{+i} \beta_{+j}-\frac{1}{1-\hat{n} \cdot \vec{\beta}_{-}} \frac{1}{\sqrt{1-\vec{\beta}_{-}^{2}}} \beta_{-i} \beta_{-j}\right] \\
& -i m \ln \omega^{-1} \epsilon^{i j}\left[C_{+} \frac{1}{1-\hat{n} \cdot \vec{\beta}_{+}} \frac{1}{\sqrt{1-\vec{\beta}_{+}^{2}}} \beta_{+i} \beta_{+j}-C_{-} \frac{1}{1-\hat{n} \cdot \vec{\beta}_{-}} \frac{1}{\sqrt{1-\vec{\beta}_{-}^{2}}} \beta_{-i} \beta_{-j}\right] \\
& -i m \frac{M}{4 \pi} \ln (R \omega) \epsilon^{i j}\left[\frac{1}{1-\hat{n} \cdot \vec{\beta}_{+}} \frac{1}{\sqrt{1-\vec{\beta}_{+}^{2}}} \beta_{+i} \beta_{+j}-\frac{1}{1-\hat{n} \cdot \vec{\beta}_{-}} \frac{1}{\sqrt{1-\vec{\beta}_{-}^{2}}} \beta_{-i} \beta_{-j}\right]
\end{aligned}
$$

The first two lines are now in complete agreement with the soft factor in Eq. (3.13) and the last line of Eq. (3.56) has the phase correction given in Eq. (3.18).

\section{Memory and tail effects from soft factors}

We have verified that the perturbation solutions in Sec. II provide the predicted soft factors resulting from the classical limit of photon and graviton soft theorems. As mentioned, the photon and graviton soft factor contributions considered in this section are universal. In addition, the soft factor results in the previous subsections involved an independent sum over the incoming and outgoing state of the probe particle. Hence the soft factor results in Eqs. (3.56) and (3.38) can be generalized to the case where we only consider light particles in the outgoing state and no light particles in the incoming state.

This was the approach taken in [72], where the graviton soft factor resulting from the probe scattering on the $D=4$ Schwarzschild spacetime [71] was generalized to the case of several light particles in the outgoing state and no incoming light particles. With this assumption on light particle states, their analysis considered the effect of the overall phase and logarithmic terms in the soft factor on the expression for the transverse and traceless components of gravitational waves. It was demonstrated that memory effect in gravitational waves now involves a tail, with contributions arising from the logarithmic terms and phase correction present in the subleading soft factor. The resulting solution in frequency space takes the form

$$
\tilde{e}_{i j}^{T T}(\omega, \vec{x})=i \omega^{-1} A_{i j}+\ln \omega^{-1} B_{i j}+\text { finite, }
$$

where $A_{i j}$ is the leading memory contribution and $B_{i j}$ is the subleading tail term with expressions

$$
\begin{aligned}
A_{i j} & =\frac{2 G}{R} \sum_{a} m_{a} \frac{1}{1-\hat{n} \cdot \vec{\beta}_{a}} \frac{1}{\sqrt{1-\vec{\beta}_{a}^{2}}}\left(\beta_{a i} \beta_{a j}\right)^{T T} \\
B_{i j}= & \frac{2 G^{2} M}{R} \sum_{a} m_{a} \frac{1}{1-\hat{n} \cdot \vec{\beta}_{a}} \frac{1}{\sqrt{1-\vec{\beta}_{a}^{2}}} \frac{1-3 \vec{\beta}_{a}^{2}+2\left|\vec{\beta}_{a}\right|^{3}}{\left|\vec{\beta}_{a}\right|^{3}} \\
& \times\left(\beta_{a i} \beta_{a j}\right)^{T T} .
\end{aligned}
$$

In Eqs. (3.58) and (3.59), the sum over all outgoing soft particles is denoted by " $a$ " and the superscript " $T T$ " refers to the transverse and traceless components. In addition, the dependence on Newton's constant $G$ has been made 
explicit which reveals that the leading memory contribution $A_{i j}$ dominates the subleading tail term $B_{i j}$.

The above result and the scattering approximation involved are realized in events where the total mass of the heavy scatterer before and after the scattering event are comparable, and far greater than the energy of the emitted light particles. Examples of such processes in particular involve binary black hole and neutron star mergers. The emitted light particles can be considered in the ultrarelativistic limit $\left|\vec{\beta}_{a}\right| \rightarrow 1$. A strict massless limit of the outgoing light states, i.e., $m_{a} \rightarrow 0$, cannot be taken if the results are based on the probe-scatterer approximation as considered in this paper and in [4]. Nevertheless, the result in the ultrarelativistic limit can be used to infer implications for gravitational waves emitted from binary black hole events. As can be noted from Eq. (3.59), in this limit $B_{i j} \rightarrow 0$ and there exists no nonlinear tail effect.

We will now directly make use of the soft factor expressions for $S_{\mathrm{gr}}$ in Eq. (3.56) and $S_{\mathrm{em}}$ in Eq. (3.38) to determine their implications on the memory and tail terms. We will reinstate the dependence on $G$ in these equations and throughout this subsection. The soft factor results in the probe-scatterer approximation can be generalized to the case where there only exist several light particles in the outgoing state. Expressing $\ln (R \omega)=$ $\ln R-\ln \omega^{-1}$, from $S_{\mathrm{gr}}$ in Eq. (3.56) we find

$$
\begin{aligned}
& S_{\mathrm{gr}}^{\stackrel{\text { single outgoing probe }}{\longrightarrow}}-8 \pi G \frac{m}{\omega} \epsilon^{i j}\left(\frac{1}{1-\hat{n} \cdot \vec{\beta}_{+}} \frac{1}{\sqrt{1-\vec{\beta}_{+}^{2}}} \beta_{+i} \beta_{+j}\right) \\
& \quad-i 8 \pi G m \ln \omega^{-1} \epsilon^{i j}\left[\left(C_{+}-2 G M\right) \frac{1}{1-\hat{n} \cdot \vec{\beta}_{+}} \frac{1}{\sqrt{1-\vec{\beta}_{+}^{2}}} \beta_{+i} \beta_{+j}\right]+\text { finite } \\
& \stackrel{\text { light outgoing particles }}{\longrightarrow}-8 \pi G\left(\frac{\epsilon^{i j}}{\omega} \sum_{a} m_{a} \frac{1}{1-\hat{n} \cdot \vec{\beta}_{a}} \frac{1}{\sqrt{1-\vec{\beta}_{a}^{2}}} \beta_{a i} \beta_{a j}\right) \\
& \quad-8 \pi G\left(i \ln \omega^{-1} \epsilon^{i j} \sum_{a} m_{a}\left(C_{a}-2 G M\right) \frac{1}{1-\hat{n} \cdot \vec{\beta}_{a}} \frac{1}{\sqrt{1-\vec{\beta}_{a}^{2}}} \beta_{a i} \beta_{a j}\right)+\text { finite. }
\end{aligned}
$$

In the first generalization of Eq. (3.60), we ignored the incoming state contribution present in Eq. (3.56), while in the second generalization we changed the outgoing state into a sum over several light particles. Denoting the result for the graviton soft factor in this case by $\widetilde{S}_{\text {gr }}$, we have

$$
\begin{aligned}
\tilde{S}_{\mathrm{gr}}= & -8 \pi G\left(\frac{\epsilon^{i j}}{\omega} \sum_{a} m_{a} \frac{1}{1-\hat{n} \cdot \vec{\beta}_{a}} \frac{1}{\sqrt{1-\vec{\beta}_{a}^{2}}} \beta_{a i} \beta_{a j}\right) \\
& -8 \pi G\left(i \ln \omega^{-1} \epsilon^{i j} \sum_{a} m_{a}\left(C_{a}-2 G M\right) \frac{1}{1-\hat{n} \cdot \vec{\beta}_{a}} \frac{1}{\sqrt{1-\vec{\beta}_{a}^{2}}} \beta_{a i} \beta_{a j}\right)+\text { finite. }
\end{aligned}
$$

Likewise, by repeating the above steps for the photon soft factor $S_{\text {em }}$ in Eq. (3.38), we find the following expression for $\tilde{S}_{\text {em }}$ : the photon soft factor in the case of there existing only multiple light particles in the final state

$$
\tilde{S}_{\mathrm{em}}=-\left(\frac{\epsilon^{i}}{\omega} \sum_{a} q_{a} \frac{1}{1-\hat{n}_{\vec{\beta}}} \beta_{a i}\right)-\left(i \ln \omega^{-1} \epsilon^{i} \sum_{a} q_{a}\left(C_{a}-2 G M\right) \frac{1}{1-\hat{n} \cdot \vec{\beta}_{a}} \beta_{a i}\right)+\text { finite }
$$

The expression for $C_{a}$, for an individual outgoing light particle follows from the expression for $C_{+}$in Eq. (3.55) with "+" replaced with " $a$ ",

$$
C_{a}=\frac{G M}{\left|\vec{\beta}_{a}\right|^{3}}\left(3 \vec{\beta}_{a}^{2}-1\right)-\frac{q_{a} Q}{4 \pi m_{a}\left|\vec{\beta}_{a}\right|^{3}}\left(1-\vec{\beta}_{a}^{2}\right)^{3 / 2}
$$

Following the analysis of [72], we now substitute Eq. (3.63) in Eq. (3.61) and Eq. (3.62) to express the graviton and photon soft factors in the following way:

$$
\begin{aligned}
\tilde{S}_{\mathrm{gr}} & =i \epsilon^{i j}\left[i \omega^{-1} \tilde{A}_{i j}^{\mathrm{gr}}+\ln \omega^{-1} \tilde{B}_{i j}^{\mathrm{gr}}\right]+\text { finite } \\
\tilde{S}_{\mathrm{em}} & =i \epsilon^{i}\left[i \omega^{-1} \tilde{A}_{i}^{\mathrm{em}}+\ln \omega^{-1} \tilde{B}_{i}^{\mathrm{em}}\right]+\text { finite }
\end{aligned}
$$


where

$$
\begin{aligned}
& \tilde{A}_{i j}^{\mathrm{gr}}=8 \pi G \sum_{a} m_{a} \frac{1}{1-\hat{n} \cdot \vec{\beta}_{a}} \frac{1}{\sqrt{1-\vec{\beta}_{a}^{2}}} \beta_{a i} \beta_{a j},
\end{aligned}
$$

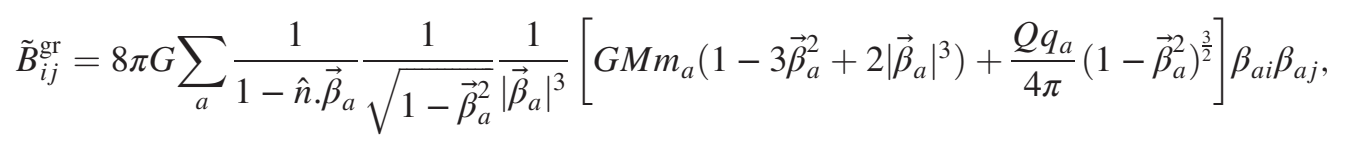

$$
\begin{aligned}
& \tilde{A}_{i}^{\mathrm{em}}=\sum_{a} q_{a} \frac{1}{1-\hat{n} \cdot \vec{\beta}_{a}} \beta_{a i}, \\
& \tilde{B}_{i}^{\text {em }}=\sum_{a} \frac{1}{1-\hat{n} \cdot \vec{\beta}_{a}} \frac{1}{\left|\vec{\beta}_{a}\right|^{3}}\left[G M q_{a}\left(1-3 \vec{\beta}_{a}^{2}+2\left|\vec{\beta}_{a}\right|^{3}\right)+\frac{Q q_{a}^{2}}{4 \pi m_{a}}\left(1-\vec{\beta}_{a}^{2}\right)^{\frac{3}{2}}\right] \beta_{a i} .
\end{aligned}
$$

Ignoring the $Q$-dependent term in Eq. (3.66), we see that the expressions in Eq. (3.65) and Eq. (3.66) agree with Eq. (3.58) and Eq. (3.59), respectively, up to a factor of $(4 \pi R)^{-1}$. Hence $\tilde{A}_{i j}^{\mathrm{gr}}$ represents the memory effect while $\tilde{B}_{i j}^{\mathrm{gr}}$ represents the corresponding tail effect of late time gravitational waves on the RN spacetime. We can similarly take $\tilde{A}_{i}^{\text {em }}$ to represent the memory effect and $\tilde{B}_{i}^{\text {em }}$ to represent the tail effect of late time electromagnetic waveforms. The results on the Schwarzschild spacetime follow from setting $Q=0$.

The $\tilde{B}_{i j}^{\mathrm{gr}}$ and $\tilde{B}_{i}^{\text {em }}$ are tails of the memory term resulting as a consequence of logarithmic and phase contributions in the subleading soft factor. In considering the $\left|\vec{\beta}_{a}\right| \rightarrow 1$ limit we see that both $\tilde{B}_{i j}^{\text {gr }} \rightarrow 0$ and $\tilde{B}_{i}^{\text {em }} \rightarrow 0$. This implies a vanishing tail in both electromagnetic and gravitational waves in the ultrarelativistic limit. Thus in the case of only massless or ultrarelativistic outgoing particles, we only have the leading memory contribution. The vanishing tail at late times is a consequence of the phase contribution to the soft factor.

For $\left|\vec{\beta}_{a}\right|<1$, the tail effect is present. If all masses and charges are comparable, we note from the dependence on $G$ in Eq. (3.66) and Eq. (3.68) that the charge $Q$ contribution will generically dominate that the mass $M$ contribution within the tail terms of gravitational and electromagnetic waves. We can also determine from the integrals evaluated in the previous subsection that the expressions for $e_{i j}(t, \vec{x})$ and $a_{i}(t, \vec{x})$ in position space which provide Eq. (3.64) will take the form

$$
\begin{aligned}
e_{i j}(t, \vec{x}) & \sim \tilde{A}_{i j}^{\mathrm{gr}}+\frac{1}{t} \tilde{B}_{i j}^{\mathrm{gr}}+\mathcal{O}\left(t^{-2}\right), \\
a_{i}(t, \vec{x}) & \sim \tilde{A}_{i}^{\mathrm{em}}+\frac{1}{t} \tilde{B}_{i}^{\mathrm{em}}+\mathcal{O}\left(t^{-2}\right) .
\end{aligned}
$$

Hence at late times, the leading memory effect will dominate the tail in both gravitational and electromagnetic waveforms.

\section{SUMMARY AND DISCUSSION}

We derived the electromagnetic and gravitational bremsstrahlung resulting from the classical scattering of a probe particle on the RN spacetime. The results were derived up to leading order in $\frac{M}{r}$ and $\frac{Q}{r}$, consistent with our assumption of a large impact parameter for the scattering. Our results in Eq. (2.72) and Eq. (2.73) for the gravitational and electromagnetic cases, respectively, can be noted as providing central charge $Q$ corrections to the known results for the gravitational radiation resulting from the probe mass scattering [4] and the electromagnetic radiation resulting from the probe charge scattering [2] on the Schwarzschild spacetime. The terms involving $Q$ in addition capture the coupling of gravitational and electromagnetic perturbations on the RN spacetime. This is noted through the specific contributions of $Q q$ in the gravitational solution Eq. (2.72) and $Q m$ in the electromagnetic solution Eq. (2.73).

The classical limit of soft theorems can be taken for scattering processes either involving large impact parameters or the probe-scatterer approximation [70]. Accordingly, in Sec. III we applied the formalism of $[70,71]$ to determine the soft factors in the probe-scatterer approximation, which includes the subleading phase corrections first described in [47] on the RN spacetime. Our result for the graviton soft factor in Eq. (3.56) and the photon soft factor in Eq. (3.38) agrees with the predicted probe-scatterer approximation expression in the presence of gravitational and electromagnetic interactions. In Sec. III D, following the approach of [72], we used the soft factor expressions to determine the late time radiation from scattering processes involving no incoming and several outgoing light particles. This extension in particular covers merger events, where the energy of the ejected light particles are less than the rest mass of the central (merged) scatterer. The memory effect in four dimensional spacetimes involves a tail contribution arising from the logarithmic terms and phase corrections present in the subleading soft factor. We expressed the photon and 
graviton soft factors derived in Eq. (3.38) and Eq. (3.56), respectively, in terms of the memory and tail effect contributions in Eq. (3.64). In all cases, the leading memory effect always dominates the tail contribution at late times, owing to the $t^{-1}$ falloff of the tail term in the radiation.

\section{ACKNOWLEDGMENTS}

We thank Alok Laddha, Arnab Priya Saha, Biswajit Sahoo, and Ashoke Sen for valuable discussions and feedback during the course of this work. K. F. would like to thank the Chennai Mathematical Institute, Chennai, India for their hospitality during the final stage of this work. K. F. was partially supported by the Max Planck Partner group 'Quantum Black Holes' between Chennai Mathematical Institute, Chennai, India and Albert Einstein Institute, Potsdam, Germany, and by a grant to the Chennai Mathematical Institute from the Infosys Foundation.

\section{APPENDIX A: SCALAR GREEN'S FUNCTION ON LINEARIZED CURVED SPACETIMES}

In flat spacetime, the scalar Green's function satisfies the equation

$\square G(x, r(\sigma))=\eta^{\mu \nu} \partial_{\mu} \partial_{\nu} G(x, r(\sigma))=-\delta^{4}(x-r(\sigma))$

which has the general solution

$$
G(x, r(\sigma))=\frac{1}{4 \pi} \delta\left(-\Omega_{0}(x, r(\sigma))\right),
$$

where

$$
\begin{aligned}
\Omega_{0}(x, r(\sigma)) & =\frac{1}{2} \eta_{\mu \nu}\left(x^{\mu}-r^{\mu}(\sigma)\right)\left(x^{\nu}-r^{\nu}(\sigma)\right) \\
& =\frac{1}{2}\left(-\left(t-r^{0}(\sigma)\right)^{2}+(\vec{x}-\vec{r}(\sigma))^{2}\right) .
\end{aligned}
$$

We may further express Eq. (A2) as a sum over retarded and advanced Green's functions

$$
\begin{aligned}
G(x, r(\sigma)) & =G_{R}(x, r(\sigma))+G_{A}(x, r(\sigma)), \\
G_{R}(x, r(\sigma)) & =\frac{1}{4 \pi R_{0}} \delta\left(t-r^{0}(\sigma)-R_{0}\right), \\
G_{A}(x, z(s)) & =\frac{1}{4 \pi r} \delta\left(t-r^{0}(\sigma)+R_{0}\right)
\end{aligned}
$$

where

$$
\vec{R}_{0}=\vec{x}-\vec{r}(\sigma), \quad R_{0}=\left|\vec{R}_{0}\right| .
$$

Hence Eq. (2.36) in flat spacetime

$$
\square \psi(x)=-\int_{-\infty}^{\infty} \delta^{4}(x-r(\sigma)) f(\sigma) d \sigma
$$

can have solutions involving only the retarded Green's function by specifying limits on the integral of the full solution

$$
\begin{aligned}
\psi(x) & =\frac{1}{4 \pi} \int_{-\infty}^{\sigma_{0}} \delta\left(-\Omega_{0}(x, r(\sigma))\right) f(\sigma) d \sigma \\
& =\frac{1}{4 \pi} \int_{-\infty}^{\infty} \frac{\delta\left(t-r^{0}(\sigma)-R_{0}\right)}{R_{0}} f(\sigma) d \sigma,
\end{aligned}
$$

where $\sigma_{0}$ is chosen such that $r^{\mu}\left(\sigma_{0}\right)$ lies outside the light cone centered on $x$. Analogous to $\Omega_{0}(x, r(\sigma))$ in flat spacetime, we can define a world function $\Omega(x, r(\sigma))$ on curved spacetimes which makes use of the geodesic distance between $x$ and $r(\sigma)$. Let $\xi^{\mu}(u)$ be the parametric solution of an unique geodesic connecting the points $x$ and $r(\sigma)$ which satisfies

$$
\frac{d U^{\alpha}}{d u}+\Gamma_{\mu \nu}^{\alpha} U^{\mu} U^{\nu}=0
$$

where $U^{\alpha}=\frac{d \xi^{\alpha}}{d u}$ and the points $x$ and $r(\sigma)$ are taken to be at the parametric values of $u_{1}$ and $u_{0}$, respectively. We then define the world function in the following way:

$$
\begin{aligned}
\Omega(x, r(\sigma)) & =\frac{u_{1}-u_{0}}{2} \int_{u_{0}}^{u_{1}} g_{\mu \nu} U^{\mu} U^{\nu} d u \\
& \equiv \frac{\left(u_{1}-u_{0}\right)^{2}}{2} g_{\mu \nu} U^{\mu} U^{\nu},
\end{aligned}
$$

where the last equality follows from the fact that since $U^{\mu}$ satisfies Eq. (A8), the integrand in the second equality of Eq. (A9) is constant. From Eq. (A9) we can derive the following relations:

$\Omega(x, r(\sigma))_{; \mu}=\left(u_{1}-u_{0}\right) U_{\mu}, \quad \Omega_{; \mu} \Omega_{;}{ }^{\mu}=2 \Omega$.

From considering the second derivatives of $\Omega$, we can also derive the following expression:

$$
\Omega(x, r(\sigma))_{; \alpha}{ }^{\alpha}=4-F(x, r(\sigma))+\mathcal{O}\left(R^{2}\right),
$$

where

$$
F(x, r(\sigma))=\frac{1}{u_{1}-u_{0}} \int_{u_{0}}^{u_{1}}\left(u-u_{0}\right)^{2} R_{\mu \nu} U^{\mu} U^{\nu} d u
$$

and $\mathcal{O}\left(R^{2}\right)$ represent terms which are quadratic and higher in curvature. As we will be seeking solutions about linearized curved backgrounds, we can ignore the $\mathcal{O}\left(R^{2}\right)$ contributions resulting from derivatives of the world function. We also note that an equivalent definition of the world function can be provided in terms of the path length 


$$
\Omega(x, r(\sigma))=-\frac{1}{2} \Delta S^{2}(x, r(\sigma)),
$$

where $\Delta S(x, r(\sigma))$ is the integral over the geodesic distance (say $s$ ) from $x$ to $r(\sigma)$

$$
\Delta S(x, r(\sigma))=\int_{r^{0}}^{t} \frac{d s}{d \xi^{0}} d \xi^{0} .
$$

Using the world function, we first assume a trial solution of Eq. (2.36) to be of the following form:

$$
\psi^{(0)}(x)=\frac{1}{4 \pi} \int_{-\infty}^{\sigma_{0}} \delta(-\Omega(x, r(\sigma))) f(\sigma) d \sigma .
$$

Using Eq. (A10) and Eq. (A11), we then find from Eq. (A15)

$$
\begin{aligned}
\psi_{; \alpha}^{(0) \alpha}= & \frac{1}{4 \pi} \int_{-\infty}^{\sigma_{0}}\left[2 \delta^{\prime \prime}(-\Omega) \Omega-4 \delta^{\prime}(-\Omega)\right. \\
& \left.+\delta^{\prime}(-\Omega) F(x, r)\right] f(\sigma) d \sigma+\mathcal{O}\left(R^{2}\right),
\end{aligned}
$$

where primes denote differentiation with respect to $-\Omega$. Reexpressing these derivatives as

$$
\delta^{\prime}(-\Omega)=\frac{d \delta(-\Omega)}{d \sigma} \frac{d \sigma}{d(-\Omega)},
$$

we find

$$
\begin{aligned}
\psi_{; \alpha}^{(0) \alpha}= & -\int_{-\infty}^{\infty} \delta^{4}(x-r(\sigma)) f(\sigma) d \sigma \\
& +\frac{1}{4 \pi} \int_{-\infty}^{\sigma_{0}} \delta^{\prime}(-\Omega) F(x, r(\sigma)) f(\sigma) d \sigma+\mathcal{O}\left(R^{2}\right) .
\end{aligned}
$$

From Eq. (A18), we can identify the following solution valid up to linear order in curvature:

$$
\begin{aligned}
\psi^{(1)}(x)= & \psi^{(0)}(x)+\delta \psi^{(0)}(x) \\
= & \psi^{(0)}(x)+\frac{1}{16 \pi^{2}} \int \sqrt{-g(y)} \delta(-\Omega(x, y)) d^{4} y \\
& \times \int_{-\infty}^{\sigma_{0}} \delta^{\prime}(-\Omega(y, r(\sigma))) F(y, r(\sigma)) f(\sigma) d \sigma .
\end{aligned}
$$

Hence $\psi^{(1)}(x)$ in Eq. (A19) does satisfy Eq. (2.36).

\section{APPENDIX B: DERIVATION OF EQS. (2.48) AND (2.49)}

1. Derivation of eq. (2.48)

The $\psi^{(0)}$ solution given in Eq. (A15) follows from the expression for the world function $\Omega(x, r(\sigma))$ and properties of the delta function.
We will derive the world function using Eq. (A13) which involves the integral $\Delta S$ over the geodesic distance $s$ from $x$ to $r(\sigma)$. From Eq. (A14), we note that this further requires us to find the expression for $\frac{d s}{d \xi^{0}}$. This can be determined from the geodesic equation

$$
\frac{d^{2} \xi^{0}}{d s^{2}}+\Gamma_{\alpha \beta}^{0} \frac{d \xi^{\alpha}}{d s} \frac{d \xi^{\beta}}{d s}=0 .
$$

Using the expression for $\Gamma_{0 k}^{0}=\phi_{, k}$, with $\phi \equiv \phi(\vec{\xi})$ along the geodesic connecting $x$ with $r(\sigma)$, we then have from Eq. (B1)

$$
\begin{aligned}
& \frac{d^{2} \xi^{0}}{d s^{2}}+2 \frac{d \phi}{d s} \frac{d \xi^{0}}{d s}=0 \\
& \Rightarrow \frac{d \xi^{0}}{d s}=A \exp [-2 \phi] \approx A(1-2 \phi)+\mathcal{O}\left(\phi^{2}\right) .
\end{aligned}
$$

Using the last line of Eq. (B2) in Eq. (A14), we find

$$
\begin{aligned}
\Delta S(x, r(\sigma)) & =\int_{r^{0}}^{t} \frac{d s}{d \xi^{0}} d \xi^{0} \approx \int_{r^{0}}^{t} \frac{1}{A}(1+2 \phi) d \xi^{0} \\
& =\frac{t-r^{0}}{A}+\frac{2}{A} \int_{r^{0}}^{t} \phi(\vec{\xi}) d \xi^{0} .
\end{aligned}
$$

We will solve the integral involved in the second line of Eq. (B3) by first parametrizing the geodesic path in the following way:

$$
\xi^{\mu}=r^{\mu}+\lambda\left(x^{\mu}-r^{\mu}\right),
$$

which is valid at the linearized level. Hence

$$
d \xi^{0}=\left(t-r^{0}\right) d \lambda,
$$

$$
\begin{aligned}
\phi(\vec{\xi}) & =-\frac{M}{8 \pi\left|\vec{r}-\lambda \vec{R}_{0}\right|} \\
& =-\frac{M}{8 \pi \sqrt{|\vec{r}|^{2}+2 \lambda R_{0}|\vec{r}| \cos \theta+\lambda^{2} R_{0}^{2}}},
\end{aligned}
$$

where $\vec{R}_{0}$ is as defined in Eq. (A5) and $\cos \theta=\frac{\vec{r} \cdot \vec{R}_{0}}{|\vec{r}| R_{0}}$. Substituting Eqs. (B5) and (B6) into the integral in the second line of Eq. (B3), we have

$$
\begin{aligned}
& \frac{2}{A} \int_{r^{0}}^{t} \phi(\vec{\xi}) d \xi^{0} \\
& \quad=-\frac{M\left(t-r^{0}\right)}{4 \pi A} \int_{0}^{1} \frac{1}{\sqrt{|\vec{r}|^{2}+2 \lambda R_{0}|\vec{r}| \cos \theta+\lambda^{2} R_{0}^{2}}} d \lambda \\
& \quad=-\frac{M\left(t-r^{0}\right)}{4 \pi R_{0} A} \ln \left(\frac{\vec{x} \cdot \vec{R}_{0}+R R_{0}}{\vec{r} \cdot \vec{R}_{0}+|\vec{r}| R_{0}}\right)=-\frac{M\left(t-r^{0}\right)}{4 \pi R_{0} A} \Gamma(\vec{x}, \vec{r})
\end{aligned}
$$


where we have replaced $|\vec{x}|=R$ and made use of the definition of $\Gamma(\vec{x}, \vec{r})$ as given in Eq. (2.50). Using Eqs. (B3) and (B7) in Eq. (A13), we then find

$$
\begin{aligned}
\Omega(x, r) & =-\frac{1}{2} \Delta S^{2}(x, r) \\
& =-\frac{\left(t-r^{0}\right)^{2}}{2 A^{2}}\left(1-\frac{M}{2 \pi R_{0}} \Gamma(\vec{x}, \vec{r})\right)+\mathcal{O}\left(\phi^{2}\right) .
\end{aligned}
$$

To complete our expression for $\Omega(x, r)$ we need to determine the constant $A^{-2}$, which follows from the normalization condition

$$
g_{\mu \nu} \frac{d \xi^{\mu}}{d s} \frac{d \xi^{\nu}}{d s}=-1
$$

Denoting $\frac{d \xi^{i}}{d \xi^{0}}=V^{i}$, Eq. (B9) can be reexpressed as

$$
A^{-2}(1+4 \phi)=(1+2 \phi)-|\vec{V}|^{2}(1-2 \phi) .
$$

We fix the constant by considering the asymptotic limit for large values of $|\vec{\xi}|$ and with $\phi \rightarrow 0$. In this case Eq. (B10) simplifies to $A^{-2}=1-\left|\vec{V}_{A}\right|^{2}$, where in denoting $|\vec{V}|$ by $\left|\vec{V}_{A}\right|$ we take this velocity to be the asymptotic value. Replacing this expression for $A^{-2}$ in Eq. (B10) we can then find the first order in $\phi$ corrections for $|\vec{V}|$. We can also take all $\phi|\vec{V}|^{2} \sim \phi$, i.e., $|\vec{V}|^{2} \approx c^{2}=1$ in all terms multiplying $\phi$, since all deviations are $\mathcal{O}\left(\phi^{2}\right)$. Accordingly, from Eq. (B10) we then find $|\vec{V}|^{2}=\left|\vec{V}_{A}\right|^{2}+4 \phi$ and hence

$$
|\vec{V}|=\left|\vec{V}_{A}\right|+2 \phi+\mathcal{O}\left(\phi^{2}\right) .
$$

The path distance $|\vec{x}-\vec{r}(\sigma)|=R_{0}$ satisfies

$$
R_{0}=\int_{r^{0}}^{t}\left|\vec{V}\left(\xi^{0}\right)\right| d \xi^{0} \approx\left|\vec{V}_{A}\right|\left(t-r^{0}\right)+2 \int_{r^{0}}^{t} \phi(\vec{\xi}) d \xi^{0},
$$

where we made use of Eq. (B11). Thus $\left|\vec{V}_{A}\right| \approx \frac{1}{t-r^{0}}\left(R_{0}-\right.$ $\left.2 \int_{r^{0}}^{t} \phi(\vec{\xi}) d \xi^{0}\right)$, which upon substituting in $A^{-2}=$ $\left(1-\left|\vec{V}_{A}\right|^{2}\right)$ gives

$$
\begin{aligned}
A^{-2}= & \frac{1}{\left(t-r^{0}\right)^{2}}\left(\left(t-r^{0}\right)^{2}-R_{0}^{2}+4 R_{0} \int_{r^{0}}^{t} \phi(\vec{\xi}) d \xi^{0}\right) \\
& +\mathcal{O}\left(\phi^{2}\right) \\
= & \frac{1}{\left(t-r^{0}\right)^{2}}\left(\left(t-r^{0}\right)^{2}-R_{0}^{2}-\frac{M R_{0}}{2 \pi} \Gamma(\vec{x}, \vec{r})\right) \\
& +\mathcal{O}\left(\phi^{2}\right),
\end{aligned}
$$

where we made use of Eq. (B7) and the property $M \frac{t-r^{0}}{R_{0}}=\frac{M}{\left|\vec{V}_{A}\right|}+\mathcal{O}\left(\phi^{2}\right) \sim M+\mathcal{O}\left(\phi^{2}\right)$. Substituting Eq. (B13) in Eq. (B8), we find

$$
\begin{aligned}
\Omega(x, r) \approx & -\frac{1}{2}\left(\left(t-r^{0}\right)^{2}-R_{0}^{2}-\frac{M R_{0}}{2 \pi} \Gamma(\vec{x}, \vec{r})\right) \\
& \times\left(1-\frac{M}{2 \pi R_{0}} \Gamma(\vec{x}, \vec{r})\right) \\
\approx & -\frac{1}{2}\left(\left(t-r^{0}\right)^{2}-\left(R_{0}+\frac{M}{4 \pi} \Gamma(\vec{x}, \vec{r})\right)^{2}\right) \\
& \times\left(1-\frac{M}{4 \pi R_{0}} \Gamma(\vec{x}, \vec{r})\right)^{2},
\end{aligned}
$$

where in the last line we could complete the squares since $\mathcal{O}\left(M^{2}\right)$ terms are ignored. Using the delta function scaling property

$$
\delta\left(\frac{y}{a}\right)=a \delta(y)
$$

where $a$ is a constant and the transformation property

$$
\delta(f(y))=\sum_{y_{0}} \frac{1}{\left|f^{\prime}\left(y_{0}\right)\right|} \delta\left(y-y_{0}\right),
$$

where $y_{0}$ is a nonrepeated root of $f(y)$, we find on substituting Eq. (B14) in Eq. (A15)

$$
\begin{aligned}
\psi^{(0)}(t, \vec{x})= & \frac{1}{4 \pi} \int_{-\infty}^{\sigma_{0}} \delta(-\Omega(x, r(\sigma))) f(\sigma) d \sigma \\
= & \frac{1}{4 \pi} \int_{-\infty}^{\infty} \frac{\delta\left(t-r^{0}-R_{0}-\frac{M}{4 \pi} \Gamma(\vec{x}, \vec{z})\right)}{R_{0}} \\
& \times f(\sigma) d \sigma+\mathcal{O}\left(M^{2}\right) .
\end{aligned}
$$

The advanced Green's function contribution is absent due to the upper limit on the integral in the first line of Eq. (B17).

\section{Derivation of eq. (2.49)}

In the case of $\delta \psi^{(0)}$, we find the following equation from Eq. (2.38):

$$
\begin{aligned}
\delta \psi^{(0)}(x)= & \frac{1}{16 \pi^{2}} \int \sqrt{-g(y)} \delta(-\Omega(x, y)) d^{4} y \\
& \times \int_{-\infty}^{\sigma_{0}} \delta^{\prime}(-\Omega(y, r(\sigma))) F(y, r(\sigma)) f(\sigma) d \sigma,
\end{aligned}
$$

with 


$$
F(y, r(\sigma))=\frac{1}{u_{1}-u_{0}} \int_{u_{0}}^{u_{1}}\left(u-u_{0}\right)^{2} R_{\mu \nu} U^{\mu} U^{\nu} d u
$$

where $U^{\alpha}=\frac{d \xi^{\alpha}}{d u}$ and the points $y$ and $r(\sigma)$ are taken to be at the parametric values of $u_{1}$ and $u_{0}$, respectively. Since we are working up to linear order in $\phi$ and the distance of $r(\sigma)$ to a black hole is taken to be large, we can approximate the black hole as a massive and charged point particle and replace $R_{\mu \nu}$ by its stress tensor. In other words, we consider

$$
R_{\mu \nu} \approx M \delta^{(3)}\left(\vec{r}^{\prime}\right) \delta_{\mu}^{0} \delta_{\nu}^{0}+\mathcal{O}\left(\phi^{2}\right)
$$

where $\vec{r}^{\prime}$ denotes the location of the black hole and $\mathcal{O}\left(\phi^{2}\right)$ involve the $\mathcal{O}\left(Q^{2}\right)$ terms in the electromagnetic stress tensor. The evaluation of Eq. (B18) also requires us to have a parametric expression for $\vec{r}^{\prime}$ in terms of $u$. For this, we consider $U^{\alpha}$ to be nearly null, i.e.,

$$
\left(U^{0}\right)^{2} \approx \vec{U}^{2}=\left(\frac{\vec{y}-\vec{r}}{u_{1}-u_{0}}\right)^{2}
$$

with the location of $\vec{r}^{\prime}$ determined from the parametric relation for a straight line path

$$
\vec{r}^{\prime}(u)=\vec{r}+\left(u-u_{0}\right) \vec{U}=\vec{r}+\left(\frac{u-u_{0}}{u_{1}-u_{0}}\right)(\vec{y}-\vec{r})
$$

Substituting Eqs. (B20)-(B22) in Eq. (B19), we find

$$
\begin{aligned}
F(y, r) & =\frac{M}{u_{1}-u_{0}} \int_{u_{0}}^{u_{1}}\left(u-u_{0}\right)^{2} \delta^{(3)}\left(\vec{r}^{\prime}(u)\right)\left(U^{0}\right)^{2} d u+\mathcal{O}\left(\phi^{2}\right) \\
& =\frac{M}{\left(u_{1}-u_{0}\right)^{3}} \int_{u_{0}}^{u_{1}}\left(u-u_{0}\right)^{2} \delta^{(3)}\left(\vec{r}^{\prime}(u)\right)(\vec{y}-\vec{r})^{2} d u+\mathcal{O}\left(\phi^{2}\right) \\
& =M \int_{u_{0}}^{u_{1}} \frac{1}{u-u_{0}} \delta^{(3)}\left(\vec{y}+\vec{r}\left(\frac{u_{1}-u}{u-u_{0}}\right)\right)(\vec{y}-\vec{r})^{2} d u+\mathcal{O}\left(\phi^{2}\right)
\end{aligned}
$$

where we made use of the delta function scaling property Eq. (B15) in the last line of Eq. (B23). For the terms other than $F$ involved in the integrand of Eq. (B18), we can use the flat spacetime expressions since Eq. (B23) is $\mathcal{O}(M)$. Thus

$$
\begin{aligned}
\int \sqrt{-g(y)} \delta(-\Omega(x, y)) d^{4} y & \rightarrow \int \delta\left(-\Omega_{0}(x, y)\right) d^{4} y \\
& =\int_{-\infty}^{\infty} \frac{1}{|\vec{x}-\vec{y}|} \delta\left(t-y^{0}-|\vec{x}-\vec{y}|\right) d^{4} y
\end{aligned}
$$

where we made use of Eq. (A7) in expressing the result as an integral over the retarded Green's function. We also change the derivative involved in the $\delta^{\prime}(-\Omega(y, r(\sigma)))$ term so that we differentiate with respect to the argument involved in the retarded Green's function

$$
\begin{aligned}
\frac{d}{d\left(-\Omega_{0}\right)} & =\frac{d\left(y^{0}-r^{0}-|\vec{y}-\vec{r}|\right)}{d\left(-\Omega_{0}\right)} \frac{d}{d\left(y^{0}-r^{0}-|\vec{y}-\vec{r}|\right)} \\
& =\frac{2}{y^{0}-r^{0}+|\vec{y}-\vec{r}|} \frac{d}{d\left(y^{0}-r^{0}-|\vec{y}-\vec{r}|\right)}
\end{aligned}
$$

Using the equation in the second line of Eq. (B25) and the property that the path from $y$ to $r(\sigma)$ is nearly null, we then have

$$
\int_{-\infty}^{\sigma_{0}} \delta^{\prime}(-\Omega(y, r(\sigma))) f(\sigma) d \sigma \rightarrow \int_{-\infty}^{\infty} \frac{1}{|\vec{y}-\vec{r}|^{2}} \delta^{\prime}\left(y^{0}-r^{0}-|\vec{y}-\vec{r}|\right) f(\sigma) d \sigma .
$$

Unless stated otherwise, $\delta^{\prime}(A)$ will always mean that we differentiate with respect to the argument of the delta function " $A$ ". Substituting Eqs. (B23), (B24), and (B26) in Eq. (B18), we get

$$
\begin{aligned}
\delta \psi^{(0)}(x)= & \frac{M}{16 \pi^{2}} \int_{-\infty}^{\infty} \frac{\delta\left(t-y^{0}-|\vec{x}-\vec{y}|\right)}{|\vec{x}-\vec{y}|} d^{4} y \int_{-\infty}^{\infty} \delta^{\prime}\left(y^{0}-r^{0}-|\vec{y}-\vec{r}|\right) f(\sigma) d \sigma \\
& \times \int_{u_{0}}^{u_{1}} \frac{1}{u-u_{0}} \delta^{(3)}\left(\vec{y}+\vec{r}\left(\frac{u_{1}-u}{u-u_{0}}\right)\right) d u .
\end{aligned}
$$


On evaluating the $d^{3} y$ integral and denoting $\alpha=\frac{u_{1}-u}{u-u_{0}}$ in Eq. (B27), we have

$$
\delta \psi^{(0)}(x)=\frac{M}{16 \pi^{2}} \int_{-\infty}^{\infty} \frac{\delta\left(t-y^{0}-|\vec{x}+\vec{r} \alpha|\right)}{|\vec{x}+\vec{r} \alpha|} d y^{0} \int_{-\infty}^{\infty} \delta^{\prime}\left(y^{0}-r^{0}-|\vec{r}(1+\alpha)|\right) f(\sigma) d \sigma \int_{u_{0}}^{u_{1}} \frac{d u}{u-u_{0}} .
$$

Changing the variable from $|\vec{r}| \alpha=v$ gives

$$
\frac{d u}{u-u_{0}}=-\frac{d v}{|\vec{r}|+v}
$$

Hence changing the variable from $u$ to $v$ in Eq. (B28), we find

$$
\delta \psi^{(0)}(x)=\frac{M}{16 \pi^{2}} \int_{0}^{\infty} d v \int_{-\infty}^{\infty} \frac{\delta\left(t-y^{0}-\rho(v)\right)}{\rho(v)(|\vec{r}|+v)} d y^{0} \int_{-\infty}^{\infty} \delta^{\prime}\left(y^{0}-r^{0}-|\vec{r}|-v\right) f(\sigma) d \sigma,
$$

where $\rho(v)$ is as defined in Eq. (2.50), i.e.,

$$
\rho(v)=\sqrt{R^{2}+v^{2}+\frac{2 v \vec{x} . \vec{r}}{|\vec{r}|}}, \quad R=|\vec{x}| .
$$

To derive Eq. (2.49) from Eq. (B30) we proceed by first making use of the shift property of the delta function to get

$$
\delta \psi^{(0)}(x)=\frac{M}{16 \pi^{2}} \int_{0}^{\infty} d v \int_{-\infty}^{\infty} \frac{\delta\left(t-y^{0}\right)}{\rho(v)(|\vec{r}|+v)} d y^{0} \int_{-\infty}^{\infty} \delta^{\prime}\left(y^{0}-r^{0}-|\vec{r}|-v-\rho(v)\right) f(\sigma) d \sigma .
$$

We now change the derivative acting on the delta function in the following way:

$$
\begin{aligned}
\delta^{\prime}\left(y^{0}-r^{0}-|\vec{r}|-v-\rho(v)\right) & =\frac{d y^{0}}{d\left(y^{0}-r^{0}-|\vec{r}|-v-\rho(v)\right)} \frac{d}{d y^{0}} \delta\left(y^{0}-r^{0}-|\vec{r}|-v-\rho(v)\right) \\
& =\frac{d}{d y^{0}} \delta\left(y^{0}-r^{0}-|\vec{r}|-v-\rho(v)\right) .
\end{aligned}
$$

Since $\rho(v)(|\vec{r}|+v)$ is independent of $y^{0}$, we thus have

$$
\delta \psi^{(0)}(x)=\frac{M}{16 \pi^{2}} \int_{-\infty}^{\infty} d y^{0} \delta\left(t-y^{0}\right) \frac{d}{d y^{0}} \int_{0}^{\infty} d v \int_{-\infty}^{\infty} \frac{\delta\left(y^{0}-r^{0}-|\vec{r}|-v-\rho(v)\right)}{\rho(v)(|\vec{r}|+v)} f(\sigma) d \sigma .
$$

Finally, by evaluating the $y^{0}$ integral, we get Eq. (2.49).

\section{APPENDIX C: EVALUATION OF INTEGRALS IN III B AND III C}

The evaluation of the integrals in the soft limit requires the asymptotic expressions for the particle trajectory and its velocity as $t \rightarrow \pm \infty$. From Eq. (3.10) we have

$$
\vec{r}=\vec{\beta}_{ \pm} t+\vec{c}_{ \pm}-C_{ \pm} \vec{\beta}_{ \pm} \ln |t|, \quad \vec{v}=\frac{d \vec{r}}{d t}=\vec{\beta}_{ \pm}\left(1-\frac{C_{ \pm}}{t}\right)
$$

where the $\mp$ signs follow the sign conventions for the incoming and outgoing particle.
Using Eq. (C1), we determine the following expressions:

$$
(1-\hat{n} \cdot \vec{v})^{-1}=\left(1-\hat{n} \cdot \vec{\beta}_{ \pm}\right)^{-1}\left[1-\frac{C_{ \pm}}{t} \frac{\hat{n} \cdot \vec{\beta}_{ \pm}}{1-\hat{n} \cdot \vec{\beta}_{ \pm}}\right]+\mathcal{O}\left(t^{-2}\right)
$$

and

$$
\phi(\vec{r}(t))=-\frac{M}{8 \pi|\vec{r}(t)|}=\mp \frac{M}{8 \pi\left|\vec{\beta}_{ \pm}\right| t}+\mathcal{O}\left(t^{-2}\right)
$$

In integrals involving $\frac{d t}{d \sigma}$, we need its asymptotic expression in the limit of large $|\vec{r}(t)|$ 


$$
\begin{aligned}
\frac{d t}{d \sigma} & =\left(-g_{\mu \nu} v^{\mu} v^{\nu}\right)^{-\frac{1}{2}}=\left[\left(1-\frac{M}{4 \pi|\vec{r}(t)|}\right)-\left(1-\frac{M}{4 \pi|\vec{r}(t)|}\right)^{-1} \vec{v}(t)^{2}\right]^{-\frac{1}{2}} \\
& =\frac{1}{\sqrt{1-\vec{v}(t)^{2}}}\left(1+\frac{M}{8 \pi|\vec{r}(t)|} \frac{1+\vec{v}(t)^{2}}{1-\vec{v}(t)^{2}}\right)+\mathcal{O}\left(\phi^{2}\right) \\
& =\frac{1}{\sqrt{1-\vec{\beta}_{ \pm}^{2}}}\left(1 \pm \frac{M}{8 \pi\left|\vec{\beta}_{ \pm}\right|} \frac{1+\vec{\beta}_{ \pm}^{2}}{1-\vec{\beta}_{ \pm}^{2}}\right)+\mathcal{O}\left(t^{-2}\right) .
\end{aligned}
$$

The integrals to be evaluated also involve terms with $\nabla_{i}$ acting on $\tilde{G}_{M}(\omega, \vec{x}, \vec{r})$ given in Eq. (3.24), where $\nabla_{i}=\frac{\partial}{\partial r^{i}}+\frac{\partial}{\partial x^{i}}$. Using Eq. (3.24), we find the following relations:

$$
\begin{aligned}
\nabla_{i} \tilde{G}_{M} & =\frac{i M}{16 \pi \omega} \frac{1}{|\vec{r}|+\hat{n} \cdot \vec{r}}\left(\frac{r_{i}}{|\vec{r}|}+\hat{n}_{i}\right) \frac{1}{R}\left\{e^{i \omega(R-\hat{n} \vec{r})}-e^{i \omega(R+|\vec{r}|)}\right\}, \\
\nabla_{i} \nabla_{j} \tilde{G}_{M}= & \frac{i M}{16 \pi \omega R}\left[i \frac{\omega}{|\vec{r}|+\hat{n} \cdot \vec{r}}\left(\frac{r_{j} \hat{n}_{i}}{|\vec{r}|}+\hat{n}_{i} \hat{n}_{j}\right)\left(e^{i \omega(R-\hat{n} \vec{r})}-e^{i \omega(R+|\vec{r}|)}\right)\right. \\
& -\frac{1}{(|\vec{r}|+\hat{n} \cdot \vec{r})^{2}}\left(\frac{r_{i}}{|\vec{r}|}+\hat{n}_{i}\right)\left(\frac{r_{j}}{|\vec{r}|}+\hat{n}_{j}\right)\left(e^{i \omega(R-\hat{n} \vec{r})}-e^{i \omega(R+|\vec{r}|)}\right) \\
& +\frac{1}{|\vec{r}|+\hat{n} \cdot \vec{r}}\left(\frac{\delta_{i j}}{|\vec{r}|}-\frac{r_{i} r_{j}}{|\vec{r}|^{3}}\right)\left(e^{i \omega(R-\hat{n} \vec{r})}-e^{i \omega(R+|\vec{r}|)}\right) \\
& \left.-i \frac{\omega}{|\vec{r}|+\hat{n} \cdot \vec{r}}\left(\frac{r_{j}}{|\vec{r}|}+\hat{n}_{j}\right)\left(\hat{n}_{i} e^{i \omega(R-\hat{n} \vec{r})}+\frac{r_{i}}{|\vec{r}|} e^{i \omega(R+|\vec{r}|)}\right)\right]
\end{aligned}
$$

and

$$
\nabla_{k} \nabla_{k} \tilde{G}_{M}=\frac{M}{8 \pi R|\vec{r}|} e^{i \omega(R+|\vec{r}|)}
$$

Using these expressions, in the following subsections we will evaluate the integrals appearing in III B and III C.

\section{Evalaution of integrals in III B}

We will first evaluate the expressions in Eqs. (3.26), (3.29), and (3.30) which contribute to the soft expression and then demonstrate that all other integrals have no terms involving $\omega^{-1}$ and $\ln \omega^{-1}$.

In the case of $\tilde{a}_{i}^{(1)}(\omega, \vec{x})$, wee see that the integrand involves a constant piece and hence requires an integration by parts [as discussed below Eq. (3.19)]. Thus Eq. (3.26) becomes

$$
\tilde{a}_{i}^{(1)}(\omega, \vec{x})=-\frac{q}{4 \pi} \frac{e^{i \omega R}}{R} \frac{1}{i \omega} \int e^{i \omega(t-\hat{n} \cdot \vec{r})} \frac{d}{d t}\left[\frac{1}{1+\phi(\vec{r})} \frac{1}{1-\hat{n} \cdot \vec{v}} v_{i}\right] d t
$$

Using Eqs. (C1)-(C3) in Eq. (C8), we find the expression

$$
\tilde{a}_{i}^{(1)}(\omega, \vec{x})=\frac{i q e^{i \omega R}}{4 \pi R} \frac{1}{\omega} \int e^{-i \omega\left(\left(\hat{n} \cdot \vec{\beta}_{ \pm}-1\right) t-C_{ \pm} \hat{n} \cdot \vec{\beta}_{ \pm} \ln |t|\right)} \frac{d}{d t}\left[\frac{\beta_{ \pm i}}{1-\hat{n} \cdot \vec{\beta}_{ \pm}}\left(1-\frac{1}{t}\left(\frac{C_{ \pm}}{1-\hat{n} \cdot \vec{\beta}_{ \pm}} \mp \frac{M}{8 \pi\left|\vec{\beta}_{ \pm}\right|}\right)\right)\right] d t+\mathcal{O}\left(t^{-3}\right) .
$$

This integral is of the $I_{1}$ type in Eq. (3.21) with the identifications 


$$
\begin{aligned}
f_{ \pm} & =\frac{i q e^{i \omega R}}{4 \pi R} \frac{\beta_{ \pm i}}{1-\hat{n} \cdot \vec{\beta}_{ \pm}} \\
k_{ \pm} & =\frac{i q e^{i \omega R}}{4 \pi R} \frac{\beta_{ \pm i}}{1-\hat{n} \cdot \vec{\beta}_{ \pm}}\left(\frac{C_{ \pm}}{\hat{n} \cdot \vec{\beta}_{ \pm}-1} \pm \frac{M}{8 \pi\left|\vec{\beta}_{ \pm}\right|}\right) \\
a_{ \pm} & =\hat{n} \cdot \vec{\beta}_{ \pm}-1, \quad b_{ \pm}=-C_{ \pm} \hat{n} \cdot \vec{\beta}_{ \pm} .
\end{aligned}
$$

Thus we get the following contribution from Eq. (C9):

$$
\begin{aligned}
\tilde{a}_{i}^{(1)}(\omega, \vec{x})= & i \frac{q}{\omega} \frac{e^{i \omega R}}{4 \pi R}\left(\frac{1}{1-\hat{n} \cdot \vec{\beta}_{+}} \beta_{+i}-\frac{1}{1-\hat{n} \cdot \vec{\beta}_{-}} \beta_{-}^{i}\right) \\
& -q \ln \omega^{-1} \frac{e^{i \omega R}}{4 \pi R}\left(\frac{C_{+}}{1-\hat{n} \cdot \vec{\beta}_{+}} \beta_{+i}-\frac{C_{-}}{1-\hat{n}_{\vec{\beta}}} \beta_{-i}-\frac{M}{8 \pi\left|\vec{\beta}_{+}\right|} \beta_{+i}-\frac{M}{8 \pi\left|\vec{\beta}_{-}\right|} \beta_{-i}\right),
\end{aligned}
$$

which is the expression given in Eq. (3.35).

As in the case of $\tilde{a}_{i}^{(1)}(\omega, \vec{x})$ in Eq. (3.26), the integral for $\tilde{a}_{i}^{(4)}(\omega, \vec{x})$ in Eq. (3.29) also requires an integration by parts, following which we have

$$
\tilde{a}_{i}^{(4)}(\omega, \vec{x})=\frac{M q e^{i \omega R}}{16 \pi^{2} R} \int d t e^{i \omega(t-\hat{n} \cdot \vec{r})} \frac{d}{d t}\left[\frac{v_{i}}{1-\hat{n} \cdot \vec{v}}\left\{\ln \frac{|\vec{r}|+\hat{n} \cdot \vec{r}}{R}+\int_{|\vec{r}|+\hat{n} \cdot \vec{r}}^{\infty} \frac{d u}{u} e^{i \omega u}\right\}\right] .
$$

Substituting Eq. (C1) in Eq. (C12), the integral takes the form of $I_{2}$ in Eq. (3.21). Hence $\tilde{a}_{i}^{(4)}(\omega, \vec{x})$ provides the expression

$$
\tilde{a}_{i}^{(4)}(\omega, \vec{x})=-M q \frac{e^{i \omega R}}{16 \pi^{2} R} \ln (\omega R)\left(\frac{1}{1-\hat{n} \cdot \vec{\beta}_{+}} \beta_{+i}-\frac{1}{1-\hat{n} \cdot \vec{\beta}_{-}} \beta_{-i}\right)+\text { finite. }
$$

This is the expression provided in Eq. (3.36).

Lastly, to evaluate $\tilde{a}_{i}^{(5)}(\omega, \vec{x})$, we use the expression in Eq. (C7) to find

$$
\tilde{a}_{i}^{(5)}(\omega, \vec{x})=-\frac{M q}{32 \pi^{2}} \frac{e^{i \omega R}}{R} \int d t \frac{v_{i}}{|\vec{r}|} e^{i \omega(t+|\vec{r}|)}
$$

On substituting Eq. (C1) in the integrand of $\tilde{a}_{i}^{(5)}(\omega, \vec{x})$ and using the expression for $I_{5}$ in Eq. (3.21), we find $\tilde{a}_{i}^{(5)}(\omega, \vec{x})=-M q \frac{e^{i \omega R}}{32 \pi^{2} R}\left(\frac{\beta_{+i}}{\left|\vec{\beta}_{+}\right|}-\frac{\beta_{-i}}{\left|\vec{\beta}_{-}\right|}\right) \ln \omega^{-1}+$ finite,

which is the expression in Eq. (3.37). These provide all the contributions to the soft factor noted in Sec. III B.

We will now demonstrate that all other integrals do not contribute in the soft limit. The integrals in $\tilde{a}_{i}^{(3)}(\omega, \vec{x})$, $\tilde{a}_{i}^{(7)}(\omega, \vec{x}), \tilde{a}_{i}^{(8)}(\omega, \vec{x})$, and $\tilde{a}_{i}^{(9)}(\omega, \vec{x})$ all involve a single derivative of $\tilde{G}_{M}$ and are all of the $I_{3}$ type in Eq. (3.21), thereby providing a finite answer with no contribution in the soft factor.

In substituting Eq. (C5) in Eq. (3.28), the resulting expression for $\tilde{a}_{i}^{(3)}(\omega, \vec{x})$ involve terms with $\hat{n}_{i}$ which do not contribute to the soft factor, since $\epsilon^{i} \hat{n}_{i}=\epsilon^{i} \frac{k_{i}}{|\vec{k}|}=0$ by Eq. (3.9). Thus the relevant part of the integral in Eq. (3.28) is

$$
\begin{aligned}
\tilde{a}_{i}^{(3)}(\omega, \vec{x})= & -\frac{M q}{16 \pi^{2}} \frac{e^{i \omega R}}{R} \\
& \times \int d t \frac{r_{i}}{|\vec{r}|(|\vec{r}|+\hat{n} . \vec{r})}\left\{e^{i \omega(t-\hat{n} \vec{r})}-e^{i \omega(t+|\vec{r}|)}\right\} \\
& + \text { terms involving } n_{i} .
\end{aligned}
$$

Substituting Eq. (C1) in Eq. (C16), the resulting integral is of the type $I_{3}$ in Eq. (3.21) with the identifications

$$
f_{ \pm}=-\frac{M q}{16 \pi^{2}} \frac{e^{i \omega R}}{R} \beta_{ \pm}^{i}, \quad k_{ \pm}=0
$$

and

$$
\begin{aligned}
& c_{ \pm}=\left|\vec{\beta}_{ \pm}\right|\left(\left|\vec{\beta}_{ \pm}\right| \pm+\hat{n} \cdot \vec{\beta}_{ \pm}\right), \quad d_{ \pm}=-C_{ \pm}\left|\vec{\beta}_{ \pm}\right|\left(\left|\vec{\beta}_{ \pm}\right| \pm+\hat{n} \cdot \vec{\beta}_{ \pm}\right), \\
& a_{ \pm}=\hat{n} \cdot \vec{\beta}_{ \pm}-1, \quad b_{ \pm}=-C_{ \pm} \hat{n} \cdot \vec{\beta}_{ \pm}, \\
& p_{ \pm}= \pm\left|\vec{\beta}_{ \pm}\right|-1, \quad q_{ \pm}=\mp C_{ \pm}\left|\vec{\beta}_{ \pm}\right| .
\end{aligned}
$$


Thus $\tilde{a}_{i}^{(3)}(\omega, \vec{x})$ does not contribute to the photon soft factor expression.

We can likewise determine that $\tilde{a}_{i}^{(7)}(\omega, \vec{x}), \tilde{a}_{i}^{(8)}(\omega, \vec{x})$, and $\tilde{a}_{i}^{(9)}(\omega, \vec{x})$ are all of type $I_{3}$. Substituting Eq. (C5) in Eqs. (3.32)-(3.34) we find the following expressions:

$$
\begin{gathered}
\tilde{a}_{i}^{(7)}(\omega, \vec{x})=\frac{Q m}{4 \pi} \frac{e^{i \omega R}}{R} \int d t \frac{d t}{d \sigma} \frac{\left(v_{i} \vec{v} \cdot \vec{r}+|\vec{r}| v_{i} \hat{n} \cdot \vec{v}\right)}{|\vec{r}|(|\vec{r}|+\hat{n} . \vec{r})}\left\{e^{i \omega(t-\hat{n} \vec{r})}-e^{i \omega(t+|\vec{r}|)}\right\}, \\
\tilde{a}_{i}^{(8)}(\omega, \vec{x})=\frac{Q m}{24 \pi} \frac{e^{i \omega R}}{R} \int d t \frac{d t}{d \sigma} \frac{r_{i}}{|\vec{r}|(|\vec{r}|+\hat{n} \cdot \vec{r})}\left\{e^{i \omega(t-\hat{n} \vec{r})}-e^{i \omega(t+|\vec{r}|)}\right\}+\text { terms involving } n_{i}, \\
\tilde{a}_{i}^{(9)}(\omega, \vec{x})=-\frac{Q m}{8 \pi} \frac{e^{i \omega R}}{R} \int d t \frac{d t}{d \sigma} \frac{v^{2} r_{i}}{|\vec{r}|(|\vec{r}|+\hat{n} \cdot \vec{r})}\left\{e^{i \omega(t-\hat{n} \vec{r})}-e^{i \omega(t+|\vec{r}|)}\right\}+\text { terms involving } n_{i},
\end{gathered}
$$

where the terms in $\hat{n}_{i}$ indicated in Eqs. (C20) and (C21) do not contribute to the soft factor. By substituting Eqs. (C1) and (C4) in Eqs. (C19)-(C21), we find integrals of the type $I_{3}$ given in Eq. (3.21) with the identifications of $a_{ \pm}, b_{ \pm}, c_{ \pm}, d_{ \pm}, p_{ \pm}$, and $q_{ \pm}$as in Eq. (C18) and with

$$
f_{ \pm}=\frac{Q m}{4 \pi} \frac{e^{i \omega R}}{R} \frac{\beta_{ \pm}^{i} \vec{\beta}_{ \pm}^{2}}{\sqrt{1-\vec{\beta}_{ \pm}^{2}}}, \quad k_{ \pm}=\frac{Q m}{4 \pi} \frac{e^{i \omega R}}{R} \frac{\beta_{ \pm}^{i} \vec{\beta}_{ \pm}^{2}}{\sqrt{1-\vec{\beta}_{ \pm}^{2}}}\left( \pm \frac{M}{8 \pi\left|\vec{\beta}_{ \pm}\right|} \frac{1+\vec{\beta}_{ \pm}^{2}}{1-\vec{\beta}_{ \pm}^{2}}-2 C_{ \pm}\right)
$$

for the expression of $\tilde{a}_{i}^{(7)}(\omega, \vec{x})$ in Eq. (C19)

$$
f_{ \pm}=\frac{Q m}{24 \pi} \frac{e^{i \omega R}}{R} \frac{\beta_{ \pm}^{i}}{\sqrt{1-\vec{\beta}_{ \pm}^{2}}}, \quad k_{ \pm}= \pm \frac{Q m}{24 \pi} \frac{e^{i \omega R}}{R} \frac{M}{8 \pi\left|\vec{\beta}_{ \pm}\right|} \frac{\beta_{ \pm}^{i}\left(1+\vec{\beta}_{ \pm}^{2}\right)}{\left(1-\vec{\beta}_{ \pm}^{2}\right)^{\frac{3}{2}}}
$$

for the expression of $\tilde{a}_{i}^{(8)}(\omega, \vec{x})$ in Eq. (C20), and

$$
f_{ \pm}=-\frac{Q m}{8 \pi} \frac{e^{i \omega R}}{R} \frac{\beta_{ \pm}^{i} \vec{\beta}_{ \pm}^{2}}{\sqrt{1-\vec{\beta}_{ \pm}^{2}}}, \quad k_{ \pm}=-\frac{Q m}{8 \pi} \frac{e^{i \omega R}}{R} \frac{\beta_{ \pm}^{i} \vec{\beta}_{ \pm}^{2}}{\sqrt{1-\vec{\beta}_{ \pm}^{2}}}\left( \pm \frac{M}{8 \pi\left|\vec{\beta}_{ \pm}\right| 1-\vec{\beta}_{ \pm}^{2}}-2 C_{ \pm}\right),
$$

for the expression of $\tilde{a}_{i}^{(9)}(\omega, \vec{x})$ in Eq. (C21). Thus the integrals $\tilde{a}_{i}^{(7)}(\omega, \vec{x}), \tilde{a}_{i}^{(8)}(\omega, \vec{x})$ and $\tilde{a}_{i}^{(9)}(\omega, \vec{x})$ do not contribute to the soft factor.

The remaining integrals $\tilde{a}_{i}^{(2)}(\omega, \vec{x})$ and $\tilde{a}_{i}^{(6)}(\omega, \vec{x})$ involve double derivatives of $\tilde{G}_{M}$ and are evaluated in an analogous manner. Using Eq. (C5) in Eq. (3.27), we express $\tilde{a}_{i}^{(2)}(\omega, \vec{x})$ as

$$
\tilde{a}_{i}^{(2)}(\omega, \vec{x})=\tilde{a}_{i, I}^{(2)}(\omega, \vec{x})+\tilde{a}_{i, I I}^{(2)}(\omega, \vec{x})+\tilde{a}_{i, I I I}^{(2)}(\omega, \vec{x})+\text { terms involving } n_{i},
$$

where

$$
\begin{gathered}
\tilde{a}_{i, I}^{(2)}(\omega, \vec{x})=-i M \frac{q}{\omega} \frac{e^{i \omega R}}{32 \pi^{2} R} \int d t\left[\frac{\vec{v} \cdot \vec{r} r_{i}}{|\vec{r}|^{2}(|r|+\hat{n} \cdot \vec{r})^{2}}+\frac{\hat{n} \cdot \vec{v} r_{i}}{|\vec{r}|(|r|+\hat{n} \cdot \vec{r})^{2}}\right]\left(e^{i \omega(t-\hat{n} \vec{r})}-e^{i \omega(t+|\vec{r}|)}\right), \\
\tilde{a}_{i, I I}^{(2)}(\omega, \vec{x})=-i M \frac{q}{\omega} \frac{e^{i \omega R}}{32 \pi^{2} R} \int d t\left[\frac{\vec{v} \cdot \vec{r} r_{i}}{|\vec{r}|^{3}(|r|+\hat{n} \cdot \vec{r})}-\frac{v_{i}}{|\vec{r}|(|r|+\hat{n} \cdot \vec{r})}\right]\left(e^{i \omega(t-\hat{n} \vec{r})}-e^{i \omega(t+|\vec{r}|)}\right), \\
\tilde{a}_{i, I I I}^{(2)}(\omega, \vec{x})=M q \frac{e^{i \omega R}}{32 \pi^{2} R} \int d t\left[\frac{\vec{v} \cdot \vec{r} r_{i}}{|\vec{r}|^{2}(|r|+\hat{n} \cdot \vec{r})}+\frac{\hat{n} \cdot \vec{v} r_{i}}{|\vec{r}|(|r|+\hat{n} \cdot \vec{r})}\right] e^{i \omega(t+|\vec{r}|)} .
\end{gathered}
$$

All terms involving $n_{i}$ were ignored in Eq. (C25), since $\epsilon^{i} n_{i}=0$ and hence does not contribute to the soft factor. Upon substituting Eq. (C1) in the above expressions, we identify that Eqs. (C26) and (C27) are integrals like $I_{4}$ and Eq. (C28) is like $I_{5}$ in Eq. (3.21), leading to the following results: 


$$
\begin{aligned}
& \tilde{a}_{i, I}^{(2)}(\omega, \vec{x})=-M q \frac{e^{i \omega R}}{32 \pi^{2} R}\left[\frac{\beta_{+i}}{\left|\vec{\beta}_{+}\right|}-\frac{\beta_{-i}}{\left|\vec{\beta}_{-}\right|}\right] \ln \omega^{-1}+\text { finite }=-\tilde{a}_{i, I I I}^{(2)}(\omega, \vec{x}), \\
& \tilde{a}_{i, I I}^{(2)}(\omega, \vec{x})=\text { finite. }
\end{aligned}
$$

Hence the expression for $\tilde{a}_{i}^{(2)}(\omega, \vec{x})$ in Eq. (C25) does not contribute to the soft factor.

As in the case $\tilde{a}_{i}^{(2)}(\omega, \vec{x})$, we express $\tilde{a}_{i}^{(6)}(\omega, \vec{x})$ as

$$
\tilde{a}_{i}^{(6)}(\omega, \vec{x})=\tilde{a}_{i, I}^{(6)}(\omega, \vec{x})+\tilde{a}_{i, I I}^{(6)}(\omega, \vec{x})+\tilde{a}_{i, I I I}^{(6)}(\omega, \vec{x})+\text { terms involving } n_{i},
$$

where

$$
\begin{gathered}
\tilde{a}_{i, I}^{(6)}(\omega, \vec{x})=i Q \frac{m}{\omega} \frac{e^{i \omega R}}{4 \pi R} \int d t \frac{d t}{d \sigma}\left[\frac{\vec{v} \cdot \vec{r} r_{i}}{|\vec{r}|^{2}(|r|+\hat{n} \cdot \vec{r})^{2}}+\frac{\hat{n} \cdot \vec{v} r_{i}}{|\vec{r}|(|r|+\hat{n} \cdot \vec{r})^{2}}\right]\left(e^{i \omega(t-\hat{n} \vec{r})}-e^{i \omega(t+|\vec{r}|)}\right), \\
\tilde{a}_{i, I I}^{(6)}(\omega, \vec{x})=i Q \frac{m}{\omega} \frac{e^{i \omega R}}{4 \pi R} \int d t \frac{d t}{d \sigma}\left[\frac{\vec{v} \cdot \vec{r} r_{i}}{|\vec{r}|^{3}(|r|+\hat{n} \cdot \vec{r})}-\frac{v_{i}}{|\vec{r}|(|r|+\hat{n} \cdot \vec{r})}\right]\left(e^{i \omega(t-\hat{n} \vec{r})}-e^{i \omega(t+|\vec{r}|)}\right) \\
\tilde{a}_{i, I I I}^{(6)}(\omega, \vec{x})=-Q m \frac{e^{i \omega R}}{4 \pi R} \int d t \frac{d t}{d \sigma}\left[\frac{\vec{v} \cdot \vec{r} r_{i}}{|\vec{r}|^{2}(|r|+\hat{n} \cdot \vec{r})}+\frac{\hat{n} \cdot \vec{v} r_{i}}{|\vec{r}|(|r|+\hat{n} \cdot \vec{r})}\right] e^{i \omega(t+|\vec{r}|)} .
\end{gathered}
$$

Using the expansions in Eqs. (C1) and (C4), with the expressions for $I_{4}$ and $I_{5}$ in Eq. (3.21), we find

$$
\begin{aligned}
& \tilde{a}_{i, I}^{(6)}(\omega, \vec{x})=Q m \frac{e^{i \omega R}}{4 \pi R}\left[\frac{\beta_{+i}}{\left|\vec{\beta}_{+}\right| \sqrt{1-\vec{\beta}_{+}^{2}}}-\frac{\beta_{-i}}{\left|\vec{\beta}_{-}\right| \sqrt{1-\vec{\beta}_{-}^{2}}}\right] \ln \omega^{-1}+\text { finite }=-\tilde{a}_{i, I I I}^{(2)}(\omega, \vec{x}), \\
& \tilde{a}_{i, I I}^{(2)}(\omega, \vec{x})=\text { finite. }
\end{aligned}
$$

Thus $\tilde{a}_{i}^{(6)}(\omega, \vec{x})$ also does not contribute to the photon soft factor.

\section{Evaluation of the integrals in III C}

The evaluation of the integrals in III C are exactly along the lines of those present in III B, which were evaluated in the previous subsection. We will thus outline the key steps leading to the results for each of the integrals.
Let us first consider the integral terms which contribute to the soft factor, i.e., $\tilde{e}_{i j}^{(1)}(\omega, \vec{x}), \tilde{e}_{i j}^{(2)}(\omega, \vec{x}), \tilde{e}_{i j}^{(3)}(\omega, \vec{x})$, and $\tilde{e}_{i j}^{(6)}(\omega, \vec{x})$. The integrands in $\tilde{e}_{i j}^{(1)}(\omega, \vec{x})$ and $\tilde{e}_{i j}^{(3)}(\omega, \vec{x})$ involve constant terms and hence require an integration by parts.

On substituting Eqs. (C1), (C3), and (C4) in Eq. (3.41), followed by an integration by parts, we find the resulting integral to be of the $I_{1}$ type in Eq. (3.21). This gives

$$
\begin{aligned}
\tilde{e}_{i j}^{(1)}(\omega, \vec{x})= & i \frac{m}{4 \pi \omega} \frac{e^{i \omega R}}{R}\left\{\frac{1}{1-\hat{n} \cdot \vec{\beta}_{+}} \frac{1}{\sqrt{1-\vec{\beta}_{+}^{2}}} \beta_{+i} \beta_{+j}-\frac{1}{1-\hat{n} \cdot \vec{\beta}_{-}} \frac{1}{\sqrt{1-\vec{\beta}_{-}^{2}}} \beta_{-i} \beta_{-j}\right\} \\
& -\frac{m}{4 \pi R} e^{i \omega R} \ln \omega^{-1}\left[\frac{1}{\sqrt{1-\vec{\beta}_{+}^{2}}} \beta_{+i} \beta_{+j}\left\{C_{+} \frac{1}{1-\hat{n} \cdot \vec{\beta}_{+}}-\frac{M_{0}}{8 \pi\left|\vec{\beta}_{+}\right|} \frac{3-\vec{\beta}_{+}^{2}}{1-\vec{\beta}_{+}^{2}}+C_{+} \frac{1}{1-\vec{\beta}_{+}^{2}}\right\}\right. \\
& \left.-\frac{1}{\sqrt{1-\vec{\beta}_{-}^{2}}} \beta_{-i} \beta_{-j}\left\{C_{-} \frac{1}{1-\hat{n} \cdot \vec{\beta}_{-}}+\frac{M_{0}}{8 \pi\left|\vec{\beta}_{-}\right|} \frac{3-\vec{\beta}_{-}^{2}}{1-\vec{\beta}_{-}^{2}}+C_{-} \frac{1}{1-\vec{\beta}_{-}^{2}}\right\}\right]
\end{aligned}
$$

which is the expression in Eq. (3.48)

In the case of $\tilde{e}_{i j}^{(3)}(\omega, \vec{x})$, we substitute Eqs. (C1) and (C4) in Eq. (3.43) and carry out an integration by parts to find an integral of the $I_{2}$ form in Eq. (3.21), leading to the result 


$$
\tilde{e}_{i j}^{(3)}(\omega, \vec{x})=-\frac{M m}{16 \pi^{2}} \ln (\omega R) \frac{e^{i \omega R}}{R}\left\{\frac{1}{1-\hat{n} \cdot \vec{\beta}_{+}} \frac{1}{\sqrt{1-\vec{\beta}_{+}^{2}}} \beta_{+i} \beta_{+j}-\frac{1}{1-\hat{n} \cdot \vec{\beta}_{-}} \frac{1}{\sqrt{1-\vec{\beta}_{-}^{2}}} \beta_{-i} \beta_{-j}\right\}
$$

in Eq. (3.50).

We will now consider the integrals for $\tilde{e}_{i j}^{(2)}(\omega, \vec{x})$ and $\tilde{e}_{i j}^{(6)}(\omega, \vec{x})$ in Eqs. (3.42) and (3.46), which are evaluated in an identical manner. In these integrals, terms involving $\delta_{i j}$ do not contribute to the soft factor as the polarization tensor satisfies $\epsilon^{i j} \delta_{i j}=\epsilon_{i}^{i}=0$ using Eq. (3.9). Furthermore, from Eq. (C6) we also note that for terms involving $\nabla_{i} \nabla_{j}$, all $\hat{n}_{i}$ or $\hat{n}_{j}$ contributions are not relevant to the soft factor due to the condition $\epsilon^{i j} n_{i}=\epsilon^{i j} \frac{k_{i}}{|\vec{k}|}=0$ in Eq. (3.9).

Thus in the case of $\tilde{e}_{i j}^{(6)}(\omega, \vec{x})$, on using Eq. (C6) in Eq. (3.46) we have

$$
\begin{aligned}
\tilde{e}_{i j}^{(6)}(\omega, \vec{x})= & Q q \frac{e^{i \omega R}}{16 \pi^{2} R} \int d t \frac{r_{i} r_{j}}{|\vec{r}|^{2}(|\vec{r}|+\hat{n} . \vec{r})} e^{i \omega(t+|\vec{r}|)} \\
& -i Q \frac{q}{\omega} \frac{e^{i \omega R}}{16 \pi^{2} R} \int d t\left[\left(\frac{r_{i} r_{j}}{(|\vec{r}|(|\vec{r}|+\hat{n} . \vec{r}))^{2}}+\frac{r_{i} r_{j}}{|\vec{r}|^{3}(|\vec{r}|+\hat{n} . \vec{r})}\right)\left(e^{i \omega(t-\hat{n} . \vec{r})}-e^{i \omega(t+|\vec{r}|)}\right)\right] \\
& + \text { terms involving } n_{i} \text { or } n_{j} .
\end{aligned}
$$

On susbstituting Eq. (C1) in Eq. (C37), we find that the integral in the first line is of the $I_{5}$ type and the integral in the second line is of the $I_{4}$ type of Eq. (3.21). Using the corresponding results for these integrals, we thus find that Eq. (C37) evaluates to

$$
\begin{aligned}
\tilde{e}_{i j}^{(6)}(\omega, \vec{x})= & Q q \frac{e^{i \omega R}}{16 \pi^{2} R} \ln \omega^{-1}\left(\frac{\beta_{+i} \beta_{+j}}{\left|\vec{\beta}_{+}\right|^{2}\left(\left|\vec{\beta}_{+}\right|+\hat{n} \vec{\beta}_{+}\right)}+\frac{\beta_{-i} \beta_{-j}}{\left|\vec{\beta}_{-}\right|^{2}\left(\left|\vec{\beta}_{-}\right|-\hat{n} \vec{\beta}_{-}\right)}\right) \\
& -Q q \frac{e^{i \omega R}}{16 \pi^{2} R} \ln \omega^{-1}\left[\left(\frac{\beta_{+i} \beta_{+j}}{\left|\vec{\beta}_{+}\right|^{2}\left(\left|\vec{\beta}_{+}\right|+\hat{n} \vec{\beta}_{+}\right)}+\frac{\beta_{-i} \beta_{-j}}{\left|\vec{\beta}_{-}\right|^{2}\left(\left|\vec{\beta}_{-}\right|-\hat{n} \vec{\beta}_{-}\right)}\right)+\frac{\beta_{+i} \beta_{+j}}{\left|\vec{\beta}_{+}\right|^{3}}+\frac{\beta_{-i} \beta_{-j}}{\left|\vec{\beta}_{-}\right|^{3}}\right]+\text { finite, }
\end{aligned}
$$

simplifying to

$$
\tilde{e}_{i j}^{(6)}(\omega, \vec{x})=-Q q \frac{e^{i \omega R}}{16 \pi^{2} R} \ln \omega^{-1}\left[\frac{\beta_{+i} \beta_{+j}}{\left|\vec{\beta}_{+}\right|^{3}}+\frac{\beta_{-i} \beta_{-j}}{\left|\vec{\beta}_{-}\right|^{3}}\right]+\text { finite, }
$$

which is the expression in Eq. (3.51). Lastly, in the case of $\tilde{e}_{i j}^{(2)}(\omega, \vec{x})$ in Eq. (3.42), we find on using Eq. (C6)

$$
\begin{aligned}
\tilde{e}_{i j}^{(2)}(\omega, \vec{x})= & M m \frac{e^{i \omega R}}{32 \pi^{2} R} \int d t \frac{d t}{d \sigma} \frac{r_{i} r_{j}}{|\vec{r}|^{2}(|\vec{r}|+\hat{n} . \vec{r})} e^{i \omega(t+|\vec{r}|)} \\
& -i M \frac{m}{\omega} \frac{e^{i \omega R}}{32 \pi^{2} R} \int d t \frac{d t}{d \sigma}\left[\left(\frac{r_{i} r_{j}}{(|\vec{r}|(|\vec{r}|+\hat{n} \cdot \vec{r}))^{2}}+\frac{r_{i} r_{j}}{|\vec{r}|^{3}(|\vec{r}|+\hat{n} \cdot \vec{r})}\right)\left(e^{i \omega(t-\hat{n} \cdot \vec{r})}-e^{i \omega(t+|\vec{r}|)}\right)\right] \\
& + \text { terms involving } n_{i} \text { or } n_{j} .
\end{aligned}
$$

The integral in first line of Eq. (C40) is of the $I_{5}$ type and the integral in the second line of Eq. (C40) is of the $I_{4}$ type given in Eq. (3.21). On substituting Eq. (C1) we thus find

$$
\tilde{e}_{i j}^{(2)}(\omega, \vec{x})=-M m \frac{e^{i \omega R}}{32 \pi^{2} R} \ln \omega^{-1}\left[\frac{\left(1+\vec{\beta}_{+}^{2}\right) \beta_{+i} \beta_{+j}}{\left|\vec{\beta}_{+}\right|^{3} \sqrt{1-\vec{\beta}_{+}^{2}}}+\frac{\left(1+\vec{\beta}_{-}^{2}\right) \beta_{-i} \beta_{-j}}{\left|\vec{\beta}_{-}\right|^{3} \sqrt{1-\vec{\beta}_{-}^{2}}}\right]+\text { finite }
$$

which is Eq. (3.49).

All remaining integrals do not contribute to the soft factor. The integral $\tilde{e}_{i j}^{(5)}(\omega, \vec{x})$ in Eq. (3.45) is proportional to $\delta_{i j}$ and hence manifestly does not contribute to the soft factor due to the condition $\epsilon^{i j} \delta_{i j}=\epsilon_{i}^{i}=0$.
The integrals for $\tilde{e}_{i j}^{(4)}(\omega, \vec{x})$ in Eq. (3.44) and $\tilde{e}_{i j}^{(7)}(\omega, \vec{x})$ in Eq. (3.47) are of a similar type. In both integrals, we substitute Eq. (C5) and can ignore all terms involving $\hat{n}_{i}$ or $\hat{n}_{j}$, since $\epsilon^{i j} n_{i}=\epsilon^{i j} \frac{k_{i}}{|\vec{k}|}=0$. Thus the relevant terms for the soft factor in Eqs. (3.44) and (3.47) on using Eq. (C5) are 


$$
\begin{gathered}
\tilde{e}_{i j}^{(4)}(\omega, \vec{x})=-M m \frac{e^{i \omega R}}{16 \pi^{2} R} \int d t \frac{d t}{d \sigma} \frac{\left(v_{i} r_{j}+v_{j} r_{i}\right)}{|\vec{r}|(|\vec{r}|+\hat{n} . \vec{r})}\left(e^{i \omega(t-\hat{n} . \vec{r})}-e^{i \omega(t+\mid \vec{r})}\right)+\text { terms involving } n_{j} \text { or } n_{i} \\
\tilde{e}_{i j}^{(7)}(\omega, \vec{x})=-Q q \frac{e^{i \omega R}}{32 \pi^{2} R} \int d t \frac{\left(v_{i} r_{j}+v_{j} r_{i}\right)}{|\vec{r}|(|\vec{r}|+\hat{n} . \vec{r})}\left(e^{i \omega(t-\hat{n} . \vec{r})}-e^{i \omega(t+|\vec{r}|)}\right)+\text { terms involving } n_{j} \text { or } n_{i} .
\end{gathered}
$$

On substituting Eqs. (C1) and (C4) in Eqs. (C42), and (C1) in Eq. (C43), we find that the above integrals are both of the $I_{3}$ type in Eq. (3.21) and hence do not contribute any $\omega^{-1}$ or $\ln \omega^{-1}$ terms.

[1] B.S. DeWitt and R.W. Brehme, Radiation damping in a gravitational field, Ann. Phys. (N.Y.) 9, 220 (1960).

[2] P. C. Peters, Electromagnetic radiation from charges in weak gravitational fields, Phys. Rev. D 7, 368 (1973).

[3] K. Westpfahl, High-speed scattering of charged and uncharged particles in general relativity, Fortschr. Phys. 33, 417 (1985).

[4] P.C. Peters, Relativistic gravitational bremsstrahlung, Phys. Rev. D 1, 1559 (1970).

[5] S. J. Kovacs and K. S. Thorne, The generation of gravitational waves. III. Derivation of bremsstrahlung formulae, Astrophys. J. 217, 252 (1977).

[6] L. Smarr, Gravitational radiation from distant encounters and from head-on collisions of black holes: The zerofrequency limit, Phys. Rev. D 15, 2069 (1977).

[7] U. Sperhake, V. Cardoso, F. Pretorius, E. Berti, and J. A. Gonzalez, High-Energy Collision of Two Black Holes, Phys. Rev. Lett. 101, 161101 (2008).

[8] W.E. East and F. Pretorius, Ultrarelativistic Black Hole Formation, Phys. Rev. Lett. 110, 101101 (2013).

[9] A. Gruzinov and G. Veneziano, Gravitational radiation from massless particle collisions, Classical Quantum Gravity 33, 125012 (2016).

[10] M. Ciafaloni, D. Colferai, F. Coradeschi, and G. Veneziano, Unified limiting form of graviton radiation at extreme energies, Phys. Rev. D 93, 044052 (2016).

[11] S. Hopper and V. Cardoso, Scattering of point particles by black holes: Gravitational radiation, Phys. Rev. D 97, 044031 (2018).

[12] S. Weinberg, Photons and gravitons in $S$-matrix theory: Derivation of charge conservation and equality of gravitational and inertial mass, Phys. Rev. 135, B1049 (1964).

[13] S. Weinberg, Infrared photons and gravitons, Phys. Rev. 140, B516 (1965).

[14] M. Gell-Mann and M. Goldberger, Scattering of low-energy photons by particles of spin 1/2, Phys. Rev. 96, 1433 (1954).

[15] F. Low, Scattering of light of very low frequency by systems of spin 1/2, Phys. Rev. 96, 1428 (1954).

[16] T. Burnett and N. M. Kroll, Extension of the Low SoftPhoton Theorem, Phys. Rev. Lett. 20, 86 (1968).

[17] A. Strominger, On BMS invariance of gravitational scattering, J. High Energy Phys. 07 (2014) 152.
[18] T. He, V. Lysov, P. Mitra, and A. Strominger, BMS supertranslations and Weinberg's soft graviton theorem, J. High Energy Phys. 05 (2015) 151.

[19] T. He, P. Mitra, A. P. Porfyriadis, and A. Strominger, New symmetries of massless QED, J. High Energy Phys. 10 (2014) 112.

[20] V. Lysov, S. Pasterski, and A. Strominger, Low's Subleading Soft Theorem as a Symmetry of QED, Phys. Rev. Lett. 113, 111601 (2014).

[21] M. Campiglia and A. Laddha, Asymptotic symmetries and subleading soft graviton theorem, Phys. Rev. D 90, 124028 (2014).

[22] D. Kapec, V. Lysov, and A. Strominger, Asymptotic symmetries of massless QED in even dimensions, Adv. Theor. Math. Phys. 21, 1747 (2017).

[23] M. Campiglia and A. Laddha, New symmetries for the gravitational S-matrix, J. High Energy Phys. 04 (2015) 076.

[24] S. G. Avery and B. U. W. Schwab, Burg-Metzner-Sachs symmetry, string theory, and soft theorems, Phys. Rev. D 93, 026003 (2016).

[25] A. Strominger, Lectures on the infrared structure of gravity and gauge theory, arXiv:1703.05448.

[26] P. Mao and J. Wu, Note on asymptotic symmetries and soft gluon theorems, Phys. Rev. D 96, 065023 (2017).

[27] A. Laddha and P. Mitra, Asymptotic symmetries and subleading soft photon theorem in effective field theories, J. High Energy Phys. 05 (2018) 132.

[28] Y. Hamada and G. Shiu, Infinite Set of Soft Theorems in Gauge-Gravity Theories as Ward-Takahashi Identities, Phys. Rev. Lett. 120, 201601 (2018).

[29] A. Ashtekar, M. Campiglia, and A. Laddha, Null infinity, the BMS group and infrared issues, Gen. Relativ. Gravit. 50, 140 (2018).

[30] F. Cachazo and A. Strominger, Evidence for a new soft graviton theorem, arXiv:1404.4091.

[31] E. Casali, Soft sub-leading divergences in Yang-Mills amplitudes, J. High Energy Phys. 08 (2014) 077.

[32] N. Afkhami-Jeddi, Soft graviton theorem in arbitrary dimensions, arXiv: 1405.3533 .

[33] B. U. W. Schwab and A. Volovich, Subleading Soft Theorem in Arbitrary Dimensions from Scattering Equations, Phys. Rev. Lett. 113, 101601 (2014).

[34] J. Broedel, M. de Leeuw, J. Plefka, and M. Rosso, Constraining subleading soft gluon and graviton theorems, Phys. Rev. D 90, 065024 (2014). 
[35] M. Zlotnikov, Sub-sub-leading soft-graviton theorem in arbitrary dimension, J. High Energy Phys. 10 (2014) 148.

[36] C. Kalousios and F. Rojas, Next to subleading soft-graviton theorem in arbitrary dimensions, J. High Energy Phys. 01 (2015) 107.

[37] H. Luo, P. Mastrolia, and W. J. Torres Bobadilla, Subleading soft behavior of QCD amplitudes, Phys. Rev. D 91, 065018 (2015).

[38] E. Conde and P. Mao, Remarks on asymptotic symmetries and the subleading soft photon theorem, Phys. Rev. D 95, 021701 (2017).

[39] P. Di Vecchia, R. Marotta, and M. Mojaza, Subsubleading soft theorems of gravitons and dilatons in the bosonic string, J. High Energy Phys. 06 (2016) 054.

[40] M. Campiglia and A. Laddha, Sub-subleading soft gravitons and large diffeomorphisms, J. High Energy Phys. 01 (2017) 036.

[41] Z. Bern, S. Davies, P. Di Vecchia, and J. Nohle, Low-energy behavior of gluons and gravitons from gauge invariance, Phys. Rev. D 90, 084035 (2014).

[42] S. He, Y. Huang, and C. Wen, Loop corrections to soft theorems in gauge theories and gravity, J. High Energy Phys. 12 (2014) 115.

[43] M. Bianchi, S. He, Y. Huang, and C. Wen, More on soft theorems: Trees, loops and strings, Phys. Rev. D 92, 065022 (2015).

[44] T. He, D. Kapec, A. Raclariu, and A. Strominger, Loopcorrected Virasaro symmetry of 4D quantum gravity, J. High Energy Phys. 08 (2017) 050.

[45] A. Sen, Subleading soft graviton theorem for loop amplitudes, J. High Energy Phys. 11 (2017) 123.

[46] N. Banerjee, S. Banerjee, S. Atul Bhatkar, and S. Jain, Conformal structure of massless scalar amplitudes beyond tree level, J. High Energy Phys. 04 (2018) 039.

[47] B. Sahoo and A. Sen, Classical and quantum results on logarithmic terms in the soft theorem in four dimensions, J. High Energy Phys. 02 (2019) 086.

[48] P. Vecchia, R. Marotta, and M. Mojaza, Multiloop soft theorem for gravitons and dilatons in the bosonic string, J. High Energy Phys. 01 (2019) 038.

[49] M. Campiglia and A. Laddha, Loop corrected soft photon theorem as a Ward identity, J. High Energy Phys. 10 (2019) 287.

[50] P. Di Vecchia, R. Marotta, and M. Mojaza, Multiloop soft theorem of the dilaton in the bosonic string, Phys. Rev. D 100, 041902 (2019).

[51] S. Atul Bhatkar, Ward identity for loop level soft photon theorem for massless QED coupled to gravity, arXiv: 1912.10229.

[52] F. Cachazo, S. He, and E. Y. Yuan, New double soft emission theorems, Phys. Rev. D 92, 065030 (2015).

[53] A. Volovich, C. Wen, and M. Zlotnikov, Double soft theorems in gauge and string theories, J. High Energy Phys. 07 (2015) 095.

[54] T. Klose, T. McLoughlin, D. Nandan, J. Plefka, and G. Travaglini, Double-soft limits of gluons and gravitons, J. High Energy Phys. 07 (2015) 135.

[55] G. Georgiou, Multi-soft theorems in gauge theory from MHV diagrams, J. High Energy Phys. 08 (2015) 128.
[56] P. Di Vecchia, R. Marotta, and M. Mojaza, Double-soft behavior for scalars and gluons from string theory, J. High Energy Phys. 12 (2015) 150.

[57] I. Low, Double soft theorems and shift symmetry in nonlinear sigma models, Phys. Rev. D 93, 045032 (2016).

[58] A. P. Saha, Double soft theorem for perturbative gravity, J. High Energy Phys. 09 (2016) 165.

[59] A. P. Saha, Double soft limit of the graviton amplitude from the Cachazo-He-Yuan formalism, Phys. Rev. D 96, 045002 (2017).

[60] S. Chakrabarti, S. P. Kashyap, B. Sahoo, A. Sen, and M. Verma, Subleading soft theorem for multiple soft gravitons, J. High Energy Phys. 12 (2017) 150.

[61] M. Zlotnikov, Leading multi-soft limits from scattering equations, J. High Energy Phys. 10 (2017) 209.

[62] S. Chakrabarti, S. P. Kashyap, B. Sahoo, A. Sen, and M. Verma, Testing subleading multiple soft graviton theorem for CHY prescription, J. High Energy Phys. 01 (2018) 090.

[63] S. Atul Bhatkar and B. Sahoo, Subleading soft theorem for arbitrary number of external soft photons and gravitons, J. High Energy Phys. 01 (2019) 153.

[64] A. Strominger and A. Zhiboedov, Gravitational memory, BMS supertranslations and soft theorems, J. High Energy Phys. 01 (2016) 086.

[65] S. Pasterski, A. Strominger, and A. Zhiboedov, New gravitational memories, J. High Energy Phys. 12 (2016) 053.

[66] S. Pasterski, Asymptotic symmetries and electromagnetic memory, J. High Energy Phys. 09 (2017) 154.

[67] P. Mao, H. Ouyang, J. Wu, and X. Wu, New electromagnetic memories and soft photon theorems, Phys. Rev. D 95, 125011 (2017).

[68] M. Pate, A. Raclariu, and A. Strominger, Color Memory: A Yang-Mills Analog of Gravitational Wave Memory, Phys. Rev. Lett. 119, 261602 (2017).

[69] Y. Hamada and S. Sugishita, Soft pion theorem, asymptotic symmetry and new memory effect, J. High Energy Phys. 11 (2017) 203.

[70] A. Laddha and A. Sen, Gravity waves from soft theorem in general dimensions, J. High Energy Phys. 09 (2018) 105.

[71] A. Laddha and A. Sen, Logarithmic terms in the soft expansion in four dimensions, J. High Energy Phys. 10 (2018) 056.

[72] A. Laddha and A. Sen, Observational signature of the logarithmic terms in the soft-graviton theorem, Phys. Rev. D 100, 024009 (2019).

[73] F. J. Zerilli, Perturbation analysis for gravitational and electromagnetic radiation in a Reissner-Nordström geometry, Phys. Rev. D 9, 860 (1974).

[74] V. Moncrief, Gauge-invariant perturbations of ReissnerNordström black holes, Phys. Rev. D 12, 1526 (1975).

[75] S. Chandrasekhar, On the equations governing the perturbations of the Reissner-Nordström black hole, Proc. R. Soc. A 365, 453 (1979).

[76] J. L. Synge, Relativity: The General Theory (North-Holland, Amsterdam, 1960).

[77] P. C. Peters, Perturbations in the Schwarzschild metric, Phys. Rev. 146, 938 (1966).

[78] A brief review of this derivation has been provided in Appendix A. 